\title{
CHIARITÉ
}

UNIVERSITÄTSMEDIZIN BERLIN

\section{Performance anxiety in professional musicians: a systematic review on prevalence, risk factors and clinical treatment effects}

I. Fernholz, J. L. M. Mumm, J. Plag, K. Noeres, G. Rotter, S. N. Willich, A. Ströhle, A. Berghöfer, A. Schmidt

\section{Document type}

Postprint (accepted version)

This version is available at

https://doi.org/10.17169/refubium-32666

\section{Citation details}

Fernholz I, Mumm JLM, Plag J, Noeres K, Rotter G, Willich SN, et al. Performance anxiety in professional musicians: a systematic review on prevalence, risk factors and clinical treatment effects. Psychological Medicine. Cambridge University Press (CUP); 2019. p. 2287-2306.

DOI: $10.1017 / \mathrm{s} 0033291719001910$

\section{Terms of use}

This work is licensed under a Creative Commons Attribution-NonCommercial-NoDerivatives 4.0 International license: https://creativecommons.org/licenses/by-nc-nd/4.0/ 


\section{Performance Anxiety in professional musicians: A systematic review on prevalence, risk factors and clinical treatment effects}

Fernholz I. ${ }^{* 1,2,3}$, Mumm J. L. M..1,2, Plag J. ${ }^{1,2}$, Noeres K. ${ }^{4}$, Rotter G. ${ }^{2,3,4}$, Willich S. N. ${ }^{2,4}$, Ströhle A. ${ }^{1}$, Berghöfer A. ${ }^{+2,4}$, Schmidt A. ${ }^{+2,3,5}$

${ }^{1}$ Charité - Universitätsmedizin Berlin, corporate member of Freie Universität Berlin, Humboldt-Universität zu Berlin, and Berlin Institute of Health, Department of Psychiatry and Psychotherapy, Campus Mitte

${ }^{2}$ Charité - Universitätsmedizin Berlin, corporate member of Freie Universität Berlin, Humboldt-Universität zu Berlin, and Berlin Institute of Health, Berlin Center for Musicians Medicine (BCMM)

${ }^{3}$ Kurt Singer Institute for Music Physiology and Musicians' Health, Hanns Eisler School of Music Berlin and University of the Arts Berlin

${ }^{4}$ Charité - Universitätsmedizin Berlin, corporate member of Freie Universität Berlin, Humboldt-Universität zu Berlin, and Berlin Institute of Health, Institute for Social Medicine, Epidemiology and Health Economics

${ }^{5}$ Charité - Universitätsmedizin Berlin, corporate member of Freie Universität Berlin, Humboldt-Universität zu Berlin, and Berlin Institute of Health, Clinic for Audiology and Phoniatrics

*authors contributed equally to this work, shared first authorship

+shared senior authorship

Conflict of interest: None

Financial support: This work was supported by grants from the Friede Springer Stiftung (for Mrs Mumm) and the Bundesministerium für Bildung und Forschung (grant number: 01PL16032) and grants from Musicboard Berlin (for Prof. Dr. Schmidt).

Corresponding Author: Prof. Dr. Alexander Schmidt, MD, MA, Berlin Center for Musicians' Medicine, Department of Audiology and Phoniatrics, Charité - Universitätsmedizin Berlin, Charitéplatz 1, 10117 Berlin, Germany, Tel: +49 30450555 125; Fax: +49 30450555 931, E-mail: alexander.schmidt@charite.de

Words: 4693 


\begin{abstract}
Music performance anxiety (MPA) is one of the most common disorders among professional musicians, nevertheless, little is known about the disease. With this systematic review prevalence, risk factors, and treatment procedures for MPA were assessed and for the first time quality assessments were carried out for all studies using standardized assessment tools. A systematic literature search was conducted via search algorithms in the databases MEDLINE, EMBASE, CINAHL, PsycArticles, Psyclnfo and ERIC. Included were case reports, case-control, cohort, cross-sectional and intervention studies examining professional musicians with MPA. For quality assessment adapted tools of the National Heart, Lung, and Blood Institute were used. A total of 43 studies were included (10 case reports, 21 intervention, 11 crosssectional, 1 cohort study). Quality ratings ranged from -11 to 6 out of a maximum of 16 points for cross-sectional /cohort studies and -4 to 11 out of 18 points for intervention studies. The prevalence of MPA was between 16.5 and $60 \%$. More women than men were affected and musicians older than 45-50 years reported less MPA than younger musicians. Regarding treatment cognitive behavioural therapy (CBT) and beta blockers were most often researched with beneficial results for CBT. However, studies with adequate control groups for CBT interventions are needed to clarify its efficacy. Studies showed methodological weaknesses, especially in the selection of participants, recording of influencing factors, blinding of interventions, randomisation of participants and analysis of comorbidity. Recommendations for further research are made.
\end{abstract}

Keywords: music performance anxiety, stage fright, social anxiety disorder, prevalence, therapy, risk factors, incidence 


\section{Introduction}

Music performance anxiety (MPA) is one of the most frequently reported disorders among musicians. The prevalence rate is estimated between 15-25\% (Spahn et al., 2011). Due to the International classification of diseases (ICD-10) (Dilling and Freyberger, 2017) it is coded as a specific phobia, in the Diagnostic and Statistical Manual of Mental Disorders (DSM-5) (American Psychiatric Association, 2013) it is classified as a subtype of social anxiety disorder (performance only subtype). A consensus on its definition has not been reached yet (Kenny, 2011).

Musicians suffering from MPA have problems in performance situations, for example in front of an audience or during orchestra rehearsals. They display physiological (most reported: tachycardia, sweating, tremor, dry mouth, shortness of breath (Hiner et al., 1987, Wesner et al., 1990)), emotional (like panic and stress) and cognitive symptoms (e.g. self-doubt or expectation of failure), often leading to avoidance (not performing) and safety behaviour (e.g. alcohol, distraction). For most musicians MPA is present directly before and during performances, while about $21 \%$ suffer from anticipatory anxiety days before the feared situation takes place (van Kemenade et al., 1995). There are varying degrees of MPA severity. Some musicians being most affected even decide to end their career. Although stress related mental disorders (like depression and anxiety disorders) are frequently observed as psychiatric comorbidities (Kenny, 2011), only about $15 \%$ of musicians affected from MPA seek help (Wesner et al., 1990). Compared to general working population musicians showed more symptoms of anxiety and depression in a Norwegian study (Vaag et al., 2016).

There are different theories regarding the etiology of anxiety disorders. Following the so called "biopsychosocial model" of anxiety disorders there are biological, psychological and social factors contributing to the development of MPA (Bandelow et al., 2017). According to Kenny (2011) a special risk factor increasing the vulnerability for MPA might be a highly demanding environment that in the same time provides little support. Besides, the exposure to early and frequent (self-) assessments in a competitive setting is seen as a specific psychological vulnerability for MPA (Kenny, 2011). 
Altogether, there are three reviews dealing with treatment options of MPA. Nagel (2010) selected studies researching cognitive behavioural therapy (CBT) and psychodynamic therapy to treat MPA and found evidence for the efficacy of CBT in MPA. However, other treatment options have not been considered. Another systematic review on treatments for MPA (professional musicians and students), describes significant positive effects on MPA and performance quality for different CBT techniques, like behavioural training, cognitive restructuring, self-instruction in combination with progressive muscle relaxation (PMR), and self-instruction in combination with attention training (Kenny, 2005). In this review only English publications were included. The third review (Brugués, 2011) particularly found beta blockers and CBT effective but declared further need for research as a conclusion. Main reasons for that were small sample sizes, no randomisation, and methodological problems. Given the fact that Nagel (2010) only focused on selected studies, Kenny (2005) restricted the review to English publications and Brugués (2011) pronounced a lack of methodological satisfactory studies, there is a need for updating the current state of research regarding MPA.

Therefore, the aim of the present systematic review was to summarize previously published literature on prevalence, risk factors and treatment effects of MPA among professional musicians respecting all languages. Furthermore, the quality of evidence was critically evaluated, to address the problem pronounced by Brugués (2011).

\section{Methods:}

The methods of the systematic literature research followed the PRISMA statement (Liberati et al., 2009, Moher et al., 2009) and the recommendations of the Cochrane Collaboration (Green and Higgins, 2011). Search methods and inclusion criteria were recorded in a protocol in advance.

\subsection{Study types}

Case reports, case-control studies, cohort studies, cross-sectional studies and intervention studies published in peer-reviewed journals were included in the review. Studies of all languages and countries of origin were considered and native speakers were recruited for all foreign-language articles. Last literature search was conducted on February 3 rd., 2018 and no time limit was set. 


\subsection{Primary outcome parameters}

Prevalence, incidence, risk factors and treatment strategies of MPA were of interest.

\subsection{Search methods}

Studies using the terms fear of performing, podium anxiety, stage fright and performance anxiety were included.

The search was carried out in two parts: an electronic and a manual search. Electronic search was conducted via search algorithms in the databases MEDLINE, EMBASE, CINAHL, PsycArticles, Psyclnfo and ERIC. Manual search included two journals: "Medical Problems of Performing Artists" and the German journal "Musikphysiologie und Musikermedizin". Complete search algorithms can be found in the appendices (appendix 1: search algorithms).

\subsection{Population and selection of studies}

Firstly, studies were selected at title, secondly, at abstract and lastly, at full text level. Therefore, pre-defined inclusion criteria were determined:

- Population:

- Musicians from at least 16 years of age

- Mixed populations with children / adolescents / adults were only included when subgroups were analysed separately. Only data of musicians from at least 16 years of age were included in the review

- Mixed populations with musicians / actors / dancers were only included when subgroups were analysed separately. Only data of musicians were included in the review

- Students at music schools, universities or conservatories

- Professional musicians as well as music teachers

- Musicians with MPA

- Outcome:

○ prevalence, incidence, risk factors and therapy

Full-text examination was carried out by a five-person team, consisting of medical staff of the Berlin Centre for Musicians' Medicine, the Institute of Social Medicine, Epidemiology and Health Economics and the Department of Psychiatry and 
Psychotherapy of the Charité - Universitätsmedizin Berlin. A consensus conference with the entire five-member team took place when inclusion of a study was ambiguous.

\subsection{Data extraction}

The following information was extracted from the studies and entered into tables sorted by study type: 1) authors, 2) publication date, 3) populations studied, 4) sample sizes of intervention group and, if applicable, control group 5) type of intervention 6) randomisation status, 7) outcomes and 8 ) results.

Regarding results, prevalence in percentages, effect sizes, correlations, mean values with standard deviations or errors, significance values, odds ratios or confidence intervals were of interest. If none of those parameters were provided, results were adopted as indicated in the particular study. With exception of percentages, no calculations were made based on the provided values.

\subsection{Quality rating}

Quality assessment tools, ensuring a standardized evaluation of studies were developed for each of the different study types (cross-sectional study, cohort study, case-control study and controlled intervention study), with exception of case reports.

For quality assessment the following instruments of the National Heart, Lung, and Blood Institute (National Heart Lung and Blood Institute, Last Updated April 2014) were used: "Quality Assessment of Controlled Intervention Studies", "Quality Assessment of Observational Cohort and Cross-Sectional Studies" and "Quality Assessment of Before-After (Pre-Post) Studies With No Control Group". To those assessment tools further elements from the quality assessment instruments of the Critical Appraisal Skills Programme (Critical Appraisal Skills Programme (CASP), 2013) and the "Methodology Checklists" of the Scottish Intercollegiate Guidelines Network (Scottish Intercollegiate Guidelines Network) were added.

A scoring system was created to systematically rate each study. Qualitative criteria were postulated dichotomously in form of "yes" or "no" questions (for example: "Was the research question or objective in this paper clearly stated?"). Questions were formulated in such a way for each inclusion criterion that a "yes" always meant that criteria were met. To obtain a final rating for each study the number of criteria rated with "no" was subtracted from the number of criteria rated with "yes". If a question was 
not applicable to a study, zero points were awarded. (see appendices 2-5) The possible overall scores differed for each evaluation instrument and thus for each study type. Controlled intervention studies could reach a maximum of 18 points, cross-sectional and cohort studies could reach a maximum of 16 points, pre-post studies without control group a maximum of 15 points and case-control studies a maximum of 14 points.

\section{Results}

The search resulted in 43 articles, comprising 21 intervention studies, 11 crosssectional studies, 1 cohort study and 10 case reports (see figure 1 for an overview of search results).

Of the intervention studies 9 comprised an active control group (Gates and Montalbo, 1987, Gates et al., 1985, James and Savage, 1984, James et al., 1977, Montello et al., 1990, Pearson and Simpson, 1978, Stanton, 1994, Sweeney and Horan, 1982, Wells et al., 2012), 9 a waiting list group or no treatment control group (Bissonnette et al., 2015, Chang et al., 2003, Khalsa and Cope, 2006, Khalsa et al., 2009, Montello et al., 1990, Nagel et al., 1989, Spahn et al., 2016, Sweeney and Horan, 1982, Valentine et al., 1995), 3 were without control group (Juncos et al., 2017, Kim, 2005, Stern et al., 2012) and 6 compared different interventions (Brodsky and Sloboda, 1997, Hinz, 2005, Khalsa et al., 2009, Kim, 2008, Sweeney and Horan, 1982, Wells et al., 2012). Some studies are listed several times because they included different kinds of control groups and / or interventions.

Quality ratings for cross-sectional studies ranged from -11 to 6 points (maximum 16 points), the cohort study reached 0 out of 16 points and intervention studies ranged from -4 to 11 points (maximum 18 points). For detailed results see tables 1-4. 


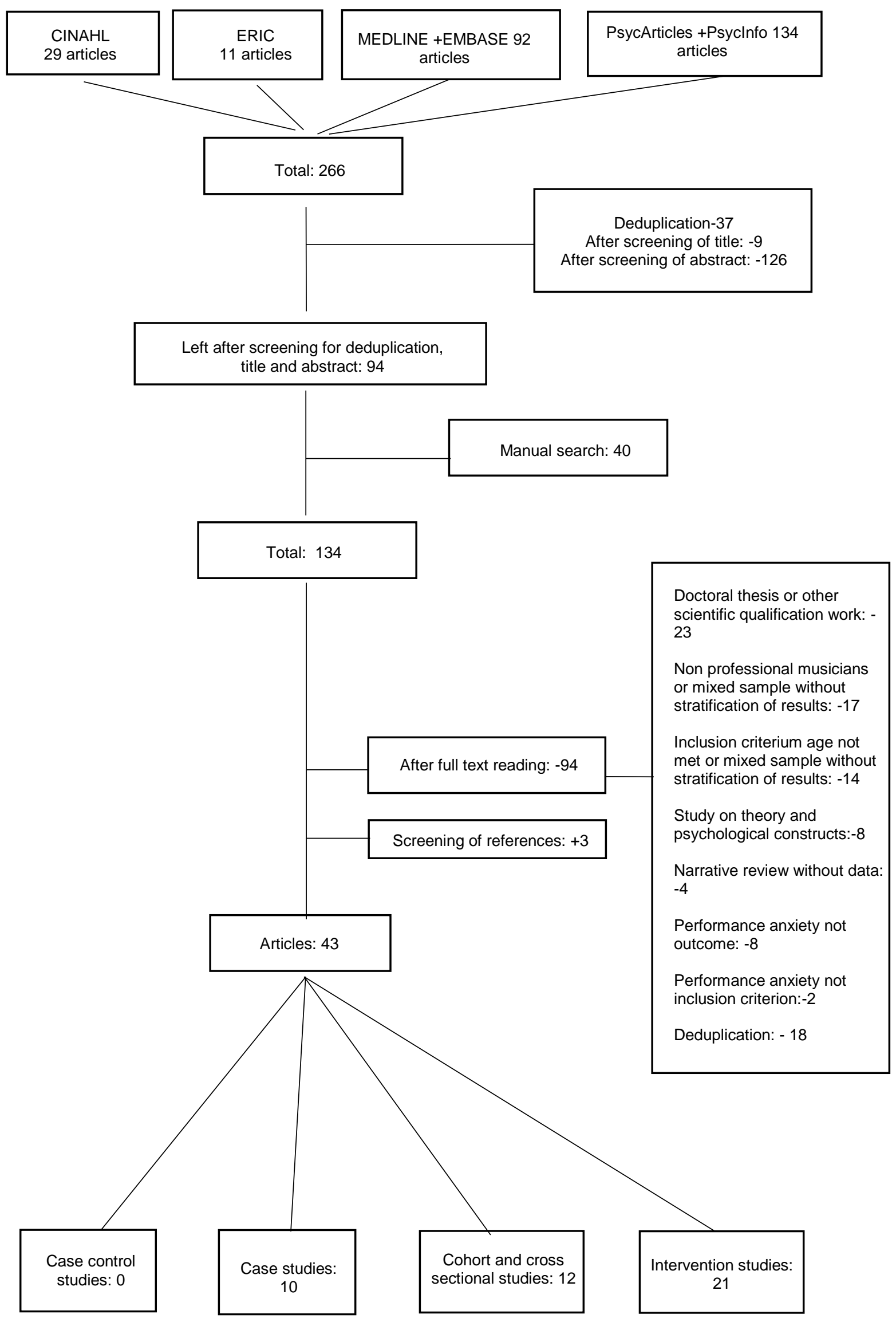

Fig 1: Overview of search and selected studies 
Table 1: Cohort study and cross-sectional studies

\begin{tabular}{|c|c|c|c|c|c|c|}
\hline Study & Population & $\mathrm{N}$ & Design & Outcome & Results & Rating \\
\hline $\begin{array}{l}\text { Hildebrandt } \\
\text { et al., } 2012\end{array}$ & $\begin{array}{l}\text { First year music students, age: } M \\
=21.3, S D=2.6,51.4 \% \text { women, } \\
48.6 \% \text { men }\end{array}$ & $\begin{array}{l}N=118 \text { at } \\
\mathrm{T} 1, N=105 \\
\mathrm{~T} 1 \text { and } \mathrm{T} 2\end{array}$ & $\begin{array}{l}\text { Cohort study, } \\
\text { T1: beginning of } \\
\text { studying music, } \\
\text { T2: after the first } \\
\text { year of studying }\end{array}$ & Risk factor: gender & $\begin{array}{l}\text { Risk factor: gender: women reported significantly more MPA than } \\
\text { men during } \mathrm{T} 1(Z=-3.67, p<.001) \text { and } \mathrm{T} 2(Z=-3.40, p=.001) .\end{array}$ & 0 \\
\hline $\begin{array}{l}\text { Fishbein et } \\
\text { al., } 1988 / \\
\text { Middlestadt, } \\
1990\end{array}$ & $\begin{array}{l}\text { Orchestral musicians, age: } M=42 \text {, } \\
36 \% \text { women, } 64 \% \text { men }\end{array}$ & $N=2212$ & $\begin{array}{l}\text { Cross-sectional } \\
\text { study }\end{array}$ & $\begin{array}{l}\text { MPA investigated with self- } \\
\text { report } \\
\text { Prevalence: presence of } \\
\text { MPA and rating of severity } \\
\text { Risk factor: gender, } \\
\text { instrument, age } \\
\text { Treatment: musicians } \\
\text { indicated undergone } \\
\text { treatments and their } \\
\text { effectivity }\end{array}$ & $\begin{array}{l}\text { Prevalence: problem: } 24 \% \text {, severe problem: } 16 \% \\
\text { Risk factor: gender: More women (19\%) than men (14\%) reported to } \\
\text { be severely affected . } \\
\text { Age: } 35-45 \text { years reporting most MPA (19\%), under } 35(17 \%) \text {, older } \\
\text { than } 45(11 \%) \text {. } \\
\text { Instrument: brass musicians more affected }(22 \%) \text {, string (14\%), } \\
\text { woodwind (14\%), other (19\%). } \\
\text { Treatment and success ratio: } \\
\text { Prescribed medication } 92 \% \text {, Aerobic exercise } 70 \% \text {, Psychological } \\
\text { counseling } 60 \%, \text { Hypnosis } 60 \% \text {, Yoga } 58 \% \text {, Alexander technique } \\
47 \%, \text { Non-prescribed medication } 46 \% \text {, Massage therapy } 38 \% \text {, See } \\
\text { general practitioner } 27 \% \text {. }\end{array}$ & -7 \\
\hline $\begin{array}{l}\text { Hiner et al., } \\
1987\end{array}$ & $\begin{array}{l}\text { Violinists taking part at a } \\
\text { competition, age: } M=24.4, S D= \\
3.2 \text {, } \\
44.8 \% \text { women, } 55.2 \% \text { men }\end{array}$ & $N=29$ & $\begin{array}{l}\text { Cross-sectional } \\
\text { study }\end{array}$ & $\begin{array}{l}\text { MPA investigated with self- } \\
\text { report } \\
\text { Treatment: rating of } \\
\text { treatment options }\end{array}$ & $\begin{array}{l}\text { Rated as effective treatment: deep breaths help relief tension } 41 \% \text {, } \\
\text { focusing intently on performance to relief tension } 69 \% \text {, medicine and } \\
\text { alcohol } 0 \% \text {. }\end{array}$ & -5 \\
\hline $\begin{array}{l}\text { Hodapp et } \\
\text { al., } 2009\end{array}$ & $\begin{array}{l}\text { Orchestra musicians (symphony } \\
\text { and opera orchestras), age: } M= \\
42.02, S D=10.08,49.18 \% \\
\text { women, } 50.82 \% \text { men, and } \\
\text { amateur orchestras, age: } M= \\
41.57, S D=14.72 \\
50 \% \text { women, } 50 \% \text { men }\end{array}$ & $\begin{array}{l}\text { Orchestras: } \\
N=122 \\
\text { Amateur } \\
\text { orchestras: } \\
N=28\end{array}$ & $\begin{array}{l}\text { Cross-sectional } \\
\text { study }\end{array}$ & $\begin{array}{l}\text { MPA investigated with TAI- } \\
\text { G, modified } \\
\text { Risk factor: neuroticism } \\
\text { (Borkenau \& Ostendorf, } \\
\text { 2008), self-efficacy } \\
\text { (Schwarzer \& Jerusalem, } \\
\text { 1999) }\end{array}$ & $\begin{array}{l}\text { Risk factor: Neuroticism and MPA: } r=.32, p<.01 \text {, self-efficacy and } \\
\text { MPA: } r=-.26, p<.05 .\end{array}$ & -1 \\
\hline $\begin{array}{l}\text { Kenny et al., } \\
2004\end{array}$ & $\begin{array}{l}\text { Operatic chorus artists, age: } M= \\
41.39, S D=9.79,65.6 \% \text { women, } \\
34.4 \% \text { men }\end{array}$ & $N=32$ & $\begin{array}{l}\text { Cross-sectional } \\
\text { study }\end{array}$ & $\begin{array}{l}\text { MPA investigated with K- } \\
\text { MPAl } \\
\text { Risk factor: gender }\end{array}$ & $\begin{array}{l}\text { Risk factor: gender: K-MPAl: no difference between men }(M=40.38 \text {, } \\
S D=26.06) \text { and women }(M=59.48, S D=35.99) .\end{array}$ & 3 \\
\hline $\begin{array}{l}\text { Kenny et al., } \\
2014\end{array}$ & $\begin{array}{l}\text { Orchestral musicians, age: } M= \\
42.1, S D=10.3,51 \% \text { female, } 49 \% \\
\text { male }\end{array}$ & $N=377$ & $\begin{array}{l}\text { Cross-sectional } \\
\text { study }\end{array}$ & $\begin{array}{l}\text { MPA investigated with K- } \\
\text { MPAI } \\
\text { Risk factors: instruments, } \\
\text { gender } \\
\text { Treatment: list of strategies }\end{array}$ & $\begin{array}{l}\text { Risk factors: gender: women sign. higher K-MPAI }(M=91.15, S D= \\
43.33) \text { than men }(M=75.95, S D=36.3), F=13.24, p=.001 \\
\text { Age: sign. gender by age interaction }(F=2.94, p=.03) \text { men: no } \\
\text { differences in K-MPAl scores between different ages, women: }<30 \\
\text { years }(M=104.5) \text { and } 41-50 \text { years }(M=99.7) \text { higher scores than } 31 \text { - } \\
40 \text { or }>50(M=78.3) \text {. } \\
\text { Instruments: no differences in MPA between instruments. } \\
\text { Treatment: } \mathrm{N}=\text { number of musicians trying the treatment, } \%= \\
\text { percent perceiving treatment effective: Beta blockers } \mathrm{N}=117,93 \% \text {, } \\
\text { increase practice } \mathrm{N}=225,91 \% \text {, mock performance practice } \mathrm{N}=170 \text {, }\end{array}$ & -1 \\
\hline
\end{tabular}




\begin{tabular}{|c|c|c|c|c|c|c|}
\hline & & & & & $\begin{array}{l}91 \% \text {, use non-prescribed medication } \mathrm{N}=15,79 \% \text {, antidepressants } \\
\mathrm{N}=14,79 \% \text {, deep breathing } \mathrm{N}=191,78 \% \text {, hypnosis } \mathrm{N}=16,76 \% \text {, } \\
\text { anxiety medications } \mathrm{N}=16,75 \% \text {, distraction methods } \mathrm{N}=43,71 \% \text {, } \\
\text { familiarize self with venue } \mathrm{N}=161,67 \% \text {, positive self-talk } \mathrm{N}=176 \text {, } \\
65 \% \text {, consult psychologist } \mathrm{N}=22,62 \% \text {, discuss with teacher } \mathrm{N}=28 \text {, } \\
60 \% \text {, consult psychiatrist } \mathrm{N}=15,54 \% \text {, discuss with partner } \mathrm{N}=116 \text {, } \\
42 \% \text {, consult doctor } \mathrm{N}=16,41 \% \text {, alcohol } \mathrm{N}=37,41 \% \text {, relaxation } \\
\text { techniques } \mathrm{N}=14012 \% \text {. }\end{array}$ & \\
\hline $\begin{array}{l}\text { Krawehl et } \\
\text { al., } 2000\end{array}$ & Music students & $N=40$ & $\begin{array}{l}\text { Cross-sectional } \\
\text { study }\end{array}$ & $\begin{array}{l}\text { MPA investigated with self- } \\
\text { report } \\
\text { Prevalence } \\
\text { Treatment }\end{array}$ & $\begin{array}{l}\text { Prevalence: } 38 \% \text { reported suffering at all performances from MPA, } \\
60 \% \text { reported suffering sometimes from MPA, } 30 \% \text { rated MPA } \\
\text { always as distressing, } 60 \% \text { rated MPA sometimes as distressing, } \\
43 \% \text { used relaxation techniques to deal with MPA. }\end{array}$ & -11 \\
\hline $\begin{array}{l}\text { Modeiros } \\
\text { Barbar et } \\
\text { al., } 2014\end{array}$ & $\begin{array}{l}\text { Musicians from musical groups, } \\
\text { schools and choirs }\end{array}$ & $N=74$ & $\begin{array}{l}\text { Cross-sectional } \\
\text { study }\end{array}$ & $\begin{array}{l}\text { MPA investigated with K- } \\
\text { MPAI } \\
\text { Prevalence }\end{array}$ & Prevalence: $39 \%$ suffer from MPA & -1 \\
\hline $\begin{array}{l}\text { Sousa et al., } \\
2016\end{array}$ & $\begin{array}{l}\text { Professional orchestra musicians } \\
\text { from } 3 \text { different orchestras, age: } M \\
=37.8, S D=9.4,33.04 \% \text { female, } \\
66.96 \% \text { male }\end{array}$ & $N=112$ & $\begin{array}{l}\text { Cross-sectional } \\
\text { study }\end{array}$ & $\begin{array}{l}\text { MPA investigated by self- } \\
\text { report in a semi-structured } \\
\text { interview } \\
\text { Prevalence }\end{array}$ & Prevalence: $21.5 \%$ suffer from MPA. & 6 \\
\hline $\begin{array}{l}\text { Steptoe \& } \\
\text { Fidler, } 1987\end{array}$ & $\begin{array}{l}\text { Orchestral players, music } \\
\text { students, amateur orchestra } \\
\text { players, age: professionals: } M= \\
37.0, S D=10.5 \text {, students: } M= \\
20.8, S D=2.2 \text {, amateurs: } M= \\
28.9, S D=14.9 \text {, altogether: } 40- \\
50 \% \text { women }\end{array}$ & $\begin{array}{l}N=106 \\
N=65 \\
\text { orchestral } \\
\text { players, } N= \\
41 \text { music } \\
\text { students, } N \\
=40 \\
\text { amateur } \\
\text { orchestra } \\
\text { players }\end{array}$ & $\begin{array}{l}\text { Cross-sectional } \\
\text { study }\end{array}$ & $\begin{array}{l}\text { MPA investigated with STAI- } \\
\text { S adapted to before } \\
\text { performance situation } \\
\text { Risk factor: age, neuroticism }\end{array}$ & $\begin{array}{l}\text { Risk factor: professionals: significant negative correlation between } \\
\text { MPA and age } r=-.35, p<.01 \rightarrow \text { less anxiety for older musicians, no } \\
\text { significant correlation between age and MPA for students }(r=.05, p \\
>.05) \text { and amateurs }(r=-.17, p>.05) \\
\text { Neuroticism significantly correlated with MPA: professionals: } r=.70 \text {, } \\
p<.01 \text {, amateurs: } r=.39, p<.05 \text {, students: } r=.31, p<.05\end{array}$ & -3 \\
\hline $\begin{array}{l}\text { Van } \\
\text { Kemenade } \\
\text { et al., } 1995\end{array}$ & $\begin{array}{l}\text { Musicians, age: } M=42.0, S D= \\
9.7,38.71 \% \text { women, } 58.71 \% \text { men, } \\
2.58 \% \text { did not indicate gender }\end{array}$ & $N=155$ & $\begin{array}{l}\text { Cross-sectional } \\
\text { study }\end{array}$ & $\begin{array}{l}\text { MPA investigated with self- } \\
\text { rating } \\
\text { Prevalence } \\
\text { Risk Factors: gender, age, } \\
\text { type of orchestra }\end{array}$ & $\begin{array}{l}\text { Prevalence: } 58.7 \% \text { experienced MPA } \\
\text { Risk factors: no differences between men and women }\left(x^{2}=1.42, p=\right. \\
.32) \text {, Type of orchestra: musicians of symphonic orchestras reported } \\
\text { more MPA ( } 62.5 \%) \text { than other musicians }(37.5 \%)\left(X^{2}=4.29, p=.04\right) \\
\text { No relationship between age and MPA }\end{array}$ & -5 \\
\hline $\begin{array}{l}\text { Wesner et } \\
\text { al., } 1990\end{array}$ & $\begin{array}{l}\text { Music students and faculty } \\
\text { members of a music school age: } \\
M=28.3, S D=10.1,51.99 \% \\
\text { women, } 45.36 \% \text { men, } 2.65 \% \text { did } \\
\text { not indicate gender }\end{array}$ & $N=302$ & $\begin{array}{l}\text { Cross-sectional } \\
\text { study }\end{array}$ & $\begin{array}{l}\text { MPA investigated with self- } \\
\text { rating } \\
\text { Prevalence } \\
\text { Risk factors: gender, age }\end{array}$ & $\begin{array}{l}\text { Prevalence: } 16.5 \% \text { report impairment due to MPA, } 21.3 \% \text { distress } \\
\text { due to MPA } \\
\text { Risk factors: age no relationship to MPA, gender: women reported } \\
\text { significantly more distress (women: } 26.8 \% \text {, men: } 16.6 \%, p=.01 \text { ) and } \\
\text { avoidance (women: } 12.7 \% \text {, men: } 5.1 \%, p=.02 \text { ) due to MPA and had } \\
\text { more often the impression of MPA having an effect on their career } \\
\text { (women: } 21.0 \% \text {, men: } 10.9 \%, p=.02 \text { ). }\end{array}$ & -7 \\
\hline
\end{tabular}

$N=$ number of participants, $M=$ mean, $S D=$ standard deviation, Outcome and results = prevalence, risk factor or treatment of MPA, rating = quality rating of study, MPA = music performance anxiety.
$M P A I=$ Kenny Music Performance Anxiety Inventory (Kenny, 2009), TAI-G = "Prüfungsängstlichkeitsinventar", modified (Brandner, 2001), STAI-S = State Anxiety Inventory (Spielberger et al., 1982). 
Table 2: Intervention studies

\begin{tabular}{|c|c|c|c|c|c|c|}
\hline Study & Population & $\mathrm{N}$ & Design & Outcome & Results & Rating \\
\hline $\begin{array}{l}\text { Bissonnette et } \\
\text { al., } 2015\end{array}$ & $\begin{array}{l}\text { Music students, } \\
\text { age: } M=21.8 \\
S D=5.258 .82 \\
\text { female, } 41.18 \\
\text { male }\end{array}$ & $\begin{array}{l}N=17, N(\text { group } \\
1)=9, N(\text { group } \\
2)=8\end{array}$ & $\begin{array}{l}\text { Intervention study } \\
(\mathrm{RCT}), \text { pre }=\mathrm{T} 1, \text { post }= \\
\mathrm{T} 2\end{array}$ & $\begin{array}{l}\text { MPA investigated with: STAI-S, PRCP, } \\
\text { quality of performance (T1 and T2) rated } \\
\text { by } 2 \text { judges } \\
\text { Treatment: group } 1: 6 \text { sessions of virtual } \\
\text { reality exposure training, each } 1 \text { hour, } \\
\text { group 2: no treatment }\end{array}$ & $\begin{array}{l}\text { PRCP: group 1: } M(\mathrm{~T} 1)=17.33, \mathrm{SE}=1.96, M(\mathrm{~T} 2)=12.11, \\
\mathrm{SE}=1.96, \text { group } 2: M(\mathrm{~T} 1)=13.13, \mathrm{SE}=2.08, M(\mathrm{~T} 2)=8.80, \\
\mathrm{SE}=2.19, \text { significant treatment effect: } F(1,15)=9.28, p< \\
.01 \text { with significant decrease in group } 1: F(1,14)=6.64, p< \\
05, \text { no significant decrease in group } 2, \text { Cohen's } d=0.15 . \\
\text { Quality of performance: group } 1: M(\mathrm{~T} 1)=78.50, S E=2.67 \text {, } \\
M(\mathrm{~T} 2)=81.39, S E=1.65, \text { group } 2: M(\mathrm{~T} 1)=77.91, S E= \\
2.84, M(\mathrm{~T} 2)=79.41, S E=1.76, \text { significant treatment effect: } \\
F(1,15)=5.77, p<.05, \text { with significant increase in group } 1: \\
F(1,15)=5.39, p<.05, \text { no significant change in group } 2 . \\
\text { STAI-S: no significant effects, separation in high and low- } \\
\text { STAI-S: high STAI-S group 1: significant decrease of STAI- } \\
\text { S: } F(1,12)=15.04, p<.01, \text { no significant change for low- } \\
\text { STAI-S anxiety group } 1 \text {, and group } 2 .\end{array}$ & 7 \\
\hline $\begin{array}{l}\text { Brodsky \& } \\
\text { Sloboda, } 1997\end{array}$ & $\begin{array}{l}\text { Professional } \\
\text { symphony } \\
\text { orchestras, age: } \\
M=36, \text { range: } \\
22-55\end{array}$ & $N=54$ & $\begin{array}{l}\text { Intervention study } \\
\text { (randomized, no } \\
\text { control group), pre = } \\
\text { T1, post = T2, follow- } \\
\text { up = T3 }\end{array}$ & $\begin{array}{l}\text { MPA investigated with: STAI-T, AMPS, } \\
\text { MPSS } \\
\text { Treatment: group 1: psychotherapeutic } \\
\text { counseling + relaxation exercise with } \\
\text { Somatron, group 2: psychotherapeutic } \\
\text { counsling + relaxation exercise with } \\
\text { music, group 3: psychotherapeutic } \\
\text { counseling + relaxation exercise } \\
\text { Relaxation = similar to PMR, } \\
\text { psychotherapeutic counseling with CBT } \\
\text { techniques, }\end{array}$ & $\begin{array}{l}\text { AMPS performer's stress: significant reduction from T1 }(M= \\
22.7) \text { to } \mathrm{T} 2(M=20.8), \text { but not from } \mathrm{T} 2 \text { to } \mathrm{T} 3(M=20.2) \text {, } \\
F(1,47)=7.42, p(\mathrm{~T} 1, \mathrm{~T} 2)=.01, p(\mathrm{~T} 2, \mathrm{~T} 3)>.05 . \\
\text { STAl-T: significant reduction of scores from } \mathrm{T} 1(M=46.4) \text { to } \\
\mathrm{T} 2(M=43.5), \text { but not from } \mathrm{T} 2 \text { to } \mathrm{T} 3(M=43.0), F(1,47)=9 \text {, } \\
p(\mathrm{~T} 1, \mathrm{~T} 2)=.004, p(\mathrm{~T} 2, \mathrm{~T} 3)>.05 . \\
\text { MPSS: } \mathrm{T} 1=60.2, \mathrm{~T} 2=62.5, \mathrm{~T} 3=59.7, \mathrm{~F}(1,46)=8.65, \\
\mathrm{p}(\mathrm{T} 1, \mathrm{~T} 2)>.05, \mathrm{p}(\mathrm{T} 2, \mathrm{~T} 3)=.005, \\
\text { MPSS no change from } \mathrm{T} 1(M=60.2) \text { to T2 }(M=62.5) \text {, but } \\
\text { significant reduction from } \mathrm{T} 2 \text { to T3 }(M=59.7), F(1,46)= \\
\text { 8.65, } p(\mathrm{~T} 1, \mathrm{~T} 2)>.05, p(\mathrm{~T} 2, \mathrm{~T} 3)=.01 . \\
\text { No significant differences between interventions on all } \\
\text { questionnaires. }\end{array}$ & 4 \\
\hline $\begin{array}{l}\text { Chang et al., } \\
2003\end{array}$ & $\begin{array}{l}\text { College and } \\
\text { graduate music } \\
\text { majors, age: } M \\
=25.1, S D= \\
6.7,74 \% \\
\text { female, } 26 \% \\
\text { male }\end{array}$ & $\begin{array}{l}N=19, N(\text { group } \\
1)=9, N(\text { group } \\
2)=10\end{array}$ & $\begin{array}{l}\text { Intervention study } \\
(\mathrm{RCT}), \text { pre }=\mathrm{T} 1 \text {, post }= \\
\mathrm{T} 2=\text { directly after a } \\
\text { concert }\end{array}$ & $\begin{array}{l}\text { MPA investigated with: PAI, STAI-S } \\
\text { Treatment: group } 1: \text { meditation class, } 8 \\
\text { weekly classes of } 1.25 \text { hours, practice } \\
\text { alone } 20 \text { minutes per day, group } 2 \text { : } \\
\text { waiting group }\end{array}$ & $\begin{array}{l}\text { PAl: group 1: } M(\mathrm{~T} 1)=47.34, S D=13.01, M(\mathrm{~T} 2)=41.64, S D \\
=14.54, t(8)=2.01, p(\mathrm{~T} 1, \mathrm{~T} 2)<.05, \text { group } 2: M(\mathrm{~T} 1)=41.40, \\
S D=6.00, M(\mathrm{~T} 2)=41.40, S D=6.42, t(9)=0.00, p(\mathrm{~T} 1, \mathrm{~T} 2)= \\
\text { 1.00. } \\
\text { STAI-S (group 1 versus group 2): group } 1 M(\mathrm{~T} 2)=35.92, S D \\
=8.18, \text { group 2: } M(\mathrm{~T} 2)=40.04, S D=8.53, t(17)=1.07, p< \\
.15, d=0.5 .\end{array}$ & 4 \\
\hline $\begin{array}{l}\text { Gates } \\
\text { \& Montalbo, } \\
1987\end{array}$ & $\begin{array}{l}\text { Singing } \\
\text { students, }\end{array}$ & $N=13$ & $\begin{array}{l}\text { Intervention study } \\
\text { (RCT, cross-over } \\
\text { study, double blind), } \\
\text { pre }=\mathrm{T} 1, \text { post }=\mathrm{T} 2 \text { (48 } \\
\text { hours later) }\end{array}$ & $\begin{array}{l}\text { MPA investigated with: } 2 \text { performances } \\
(T 1, T 2) \text { : ranking of subject's anxiety }(0= \\
\text { no nervousness, } 10=\text { highest possible } \\
\text { degree) and judges evaluate } \\
\text { performances. }\end{array}$ & $\begin{array}{l}\text { Judges ratings: } M(\text { Nadolol })=6.76, M(\text { placebo })=7.17, p= \\
.07 . \\
\text { Ranking of anxiety before performance: } M(\text { Nadolol })=3.92 \\
M(\text { placebo })=3.23, p=.73\end{array}$ & 6 \\
\hline
\end{tabular}




\begin{tabular}{|c|c|c|c|c|c|c|}
\hline & & & & $\begin{array}{l}\text { Treatment: single dose of beta-blockade } \\
\text { (20mg Nadolol) or placebo before } \\
\text { performance }\end{array}$ & & \\
\hline $\begin{array}{l}\text { Gates et al., } \\
1985\end{array}$ & $\begin{array}{l}\text { Singing } \\
\text { students, age: } \\
M=25.7, S D= \\
7.1,2264.71 \% \\
\text { female, } 35.29 \% \\
\text { male }\end{array}$ & $N=34$ & $\begin{array}{l}\text { Intervention study } \\
\text { (randomized, double } \\
\text { blind) }\end{array}$ & $\begin{array}{l}\text { MPA investigated with: } 2 \text { performances } \\
\text { (T1, T2). Self-rating of anxiety before } \\
\text { and after performances and of } \\
\text { performance quality. Rating of } \\
\text { performance quality by judges ( } 1-10,10 \\
\text { = being perfect) and heart rate. } \\
\text { Treatment: application } 3 \text { hours before } \\
\text { T1: group } 1: 0 \mathrm{mg} \text { Nadolol, group } 2: \\
\text { 20mg Nadolol, group } 3: 40 \mathrm{mg} \text { Nadolol, } \\
\text { group 4: } 80 \mathrm{mg} \text { Nadolol, all groups } \\
\text { received placebo } 3 \text { hours before T2 }\end{array}$ & $\begin{array}{l}\text { Heart rate was significantly lower for all nadolol } \\
\text { performances }(p<.001) \text {. Students' ratings of their } \\
\text { performance quality at T1 and T2 did not differ for the } 4 \\
\text { groups }(p=.49) \text {. Judges ratings of performances for nadolol } \\
\text { groups were significantly better for group } 2(p=.02) \text {. Self- } \\
\text { rated anxiety levels between the two performances }(\mathrm{T} 1, \mathrm{~T} 2) \\
\text { did not differ between the } 4 \text { groups before }(M(\text { group } 1, \mathrm{~T} 1)= \\
4.5, M \text { (group } 1, \mathrm{~T} 2)=4.8, M \text { (group } 2, \mathrm{~T} 1)=4.9, M \text { (group } 2 \text {, } \\
\mathrm{T} 2)=6.7, M \text { (group } 3, \mathrm{~T} 1)=4.9, M(\text { group } 3, \mathrm{~T} 2)=5.4 \text {, } \\
M(\text { group } 4, \mathrm{~T} 1)=5.0, M(\text { group } 4, \mathrm{~T} 2)=4.7, p=.46) \text { and } \\
\text { after }(M(\text { group } 1, \mathrm{~T} 1)=4.9, M(\text { group } 1, \mathrm{~T} 2)=4.2, M(\text { group } 2 \text {, } \\
\mathrm{T} 1)=2.4, M(\text { group } 2, \mathrm{~T} 2)=3.1, M(\text { group } 3, \mathrm{~T} 1)=3.5 \text {, } \\
M(\text { group } 3, \mathrm{~T} 2)=4.7, M(\text { group } 4, \mathrm{~T} 1)=2.3, M(\text { group } 4, \mathrm{~T} 2)= \\
2.6, p=.73) \text { the performances. }\end{array}$ & \\
\hline Hinz et al., 2005 & $\begin{array}{l}\text { Music students, } \\
\text { musicians, age: } \\
M=23.8 \text {, } \\
53.85 \% \text { female, } \\
46.15 \% \text { male }\end{array}$ & $\begin{array}{l}N=26,5 \\
\text { dropouts } \\
N(\text { Group } 1)= \\
11, N(\text { Group } 2) \\
=10\end{array}$ & $\begin{array}{l}\text { Intervention study, } \\
\text { (participants could } \\
\text { choose between } 2 \\
\text { interventions) pre = T1, } \\
\text { Post = T2 after one } \\
\text { year }\end{array}$ & $\begin{array}{l}\text { MPA investigated with: } 24 \mathrm{~h} \text { ECG on a } \\
\text { day with performance, STAI not reported } \\
\text { if state or trait version was used, POA, } \\
\text { KAB } \\
\text { Treatment: Group 1: beta blocker ( } 25 \mathrm{mg} \\
\text { Propranolol) } 1-1.5 \text { hours before } \\
\text { performance 2, Group 2: training of } \\
\text { progressive muscle relaxation } \\
\text { (Jacobsen) for a few weeks before } \\
\text { performance } 2\end{array}$ & 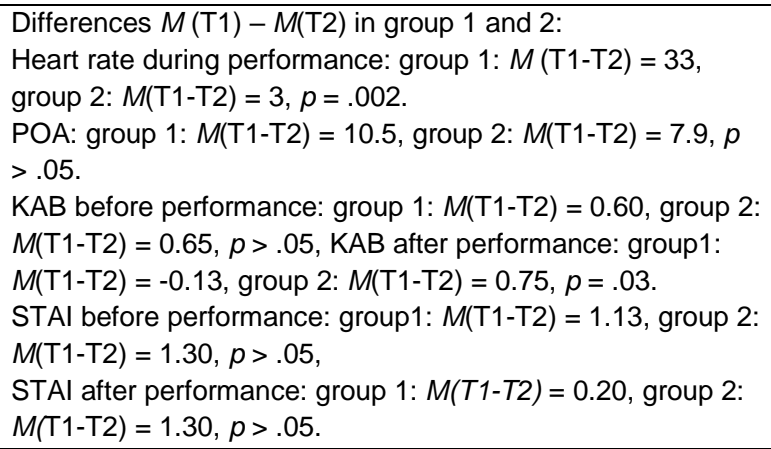 & -4 \\
\hline $\begin{array}{l}\text { James \& } \\
\text { Savage, } 1984\end{array}$ & $\begin{array}{l}\text { String players, } \\
\text { students, } \\
\text { participants had } \\
\text { no nervous } \\
\text { illness }\end{array}$ & $\begin{array}{l}N=33,2 \\
\text { dropouts }\end{array}$ & $\begin{array}{l}\text { Intervention study } \\
\text { (RCT, double blind, } \\
\text { cross-over study at day } \\
1 \text { and } 2 \text { ) }\end{array}$ & $\begin{array}{l}\text { Anxiety investigated with: } 2 \\
\text { performances of } 15 \text { minutes (day } 1 \text { and } \\
\text { day 2), } \\
\text { Ratings: musical assessment of } 5 \\
\text { musical variables from } 1=\text { poor to } \\
5=\text { excellent, observer rating of } 5 \\
\text { symptoms and self-rating of } 10 \text { physical } \\
\text { symptoms ( } 0=\text { absent, } 8=\text { very marked), } \\
\text { self-rating of physical symptoms, pulse, } \\
\text { blood pressure } \\
\text { Treatment: group } 1 \text { : Diazepam } 2 \mathrm{mg} / \\
\text { placebo, (administered } 1 \text { hour before } \\
\text { performance), group } 2 \text { : nadolol, } 40 \mathrm{mg} / \\
\text { placebo (4h before performance) }\end{array}$ & $\begin{array}{l}\text { Musical assessment: nadolol and placebo: significant better } \\
\text { bow control with nadolol (day } 1: M=15.3, S D=1.71 \text {, day } 2 \text { : } \\
M=14.8, S D=3.96 \text { ) compared to placebo (day } 1: M=13.5 \text {, } \\
S D=2.55 \text {, day } 2: M=12.7, S D=.73 \text { ) }, p<.05 \text {, no other } \\
\text { significant differences in musical assessment, diazepam and } \\
\text { placebo: no significant differences. } \\
\text { Observer ratings: nadolol and placebo: patients receiving } \\
\text { nadolol were significantly paler than those receiving placebo, } \\
\text { no other significant differences, diazepam vs. placebo: no } \\
\text { differences. } \\
\text { Self-ratings: no significant differences in physical symptoms } \\
\text { between placebo and nadolol or diazepam. } \\
\text { Blood pressure: no significant differences between placebo } \\
\text { and nadolol or diazepam. }\end{array}$ & 10 \\
\hline
\end{tabular}




\begin{tabular}{|c|c|c|c|c|c|c|}
\hline & & & & & $\begin{array}{l}\text { Pulse: nadolol group significant slower pulse rate than } \\
\text { placebo (nadolol group approximately } 15 \text { bpm slower than } \\
\text { placebo), no differences between placebo and diazepam. }\end{array}$ & \\
\hline $\begin{array}{l}\text { James et al., } \\
1977 \text { / Pearson } \\
\& \text { Simpson, } \\
1978\end{array}$ & $\begin{array}{l}\text { String players, } \\
\text { mostly } \\
\text { students, age: } \\
M=23,75 \% \\
\text { women, } 25 \% \\
\text { men, all free } \\
\text { from mental } \\
\text { illness }\end{array}$ & $N=24$ & $\begin{array}{l}\text { Intervention study, } \\
\text { (randomized, cross- } \\
\text { over study at day } 1 \text { and } \\
2 \text {, double blind) }\end{array}$ & $\begin{array}{l}\text { Anxiety investigated before } 2 \\
\text { performances with anxiety rating from } 0 \\
=\text { I feel relaxed, to } 100=I \text { feel petrified } \\
\text { and graded list }(1=\text { nonchalant, } 6= \\
\text { panicky), blood pressure, pulse } \\
\text { performance rated by } 2 \text { independent } \\
\text { experts } \\
\text { Treatment: oxprenolol } 40 \mathrm{mg} \text {, placebo } 90 \\
\text { min before performances with an } \\
\text { audience on two consecutive days }\end{array}$ & $\begin{array}{l}\text { Oxprenolol: significantly lower anxiety rating (Oxprenolol: } M \\
=46, S D=4.8 \text {, Placebo: } M=57, S D=4.0, p<.05) \text { and } \\
\text { lower graded list (Oxprenolol: } M=2.92, S D=0.24 \text {, placebo: } \\
M=3.71, S D=0.23, p<.005) \\
\text { Significantly reduced pulse-rate and systolic blood pressure } \\
\text { for oxprenolol performances. }\end{array}$ & 6 \\
\hline $\begin{array}{l}\text { Juncos et al., } \\
2017\end{array}$ & $\begin{array}{l}\text { Student } \\
\text { vocalists, age: } \\
M=23.29, \\
S D=3.73, \\
85.71 \% \text { female, } \\
14.29 \% \text { male, } \\
\text { MPA }\end{array}$ & $N=7$ & $\begin{array}{l}\text { Intervention study (no } \\
\text { control group), } \\
\text { Baseline, pre = T1, } \\
\text { post }=\mathrm{T} 2,1 \text {-month } \\
\text { follow-up }=\mathrm{T} 3,3- \\
\text { month follow-up }=\mathrm{T} 4\end{array}$ & $\begin{array}{l}\text { MPA investigated with: K-MPAl, ACQ, } \\
\text { performance at T1 and T2 with quality } \\
\text { rating due to MPQ (average rating and } \\
\text { overall rating) from } 3 \text { independent raters } \\
\text { Treatment: } 12 \text { sessions of Acceptance } \\
\text { and Commitment Therapy }\end{array}$ & $\begin{array}{l}\text { Performance quality did not change from T1 to T2: average } \\
\text { rating: } F(1,6)=0.67, p=.45 \text {, overall rating: } F(1,6)=1.05, p= \\
\text {.33. } \\
\text { K-MPAl from baseline }(M=146.71) \text { to T2 }(M=115.17): t(11) \\
=2.79, p<.05, \text { Hedges' } g=1.55 ., \mathrm{K}-\mathrm{MPAl} \text { from baseline to } \\
\text { T3 }(M=107.83): t(11)=2.89, p<.05 \text {, Hedges' } g=1.61, \mathrm{~K}- \\
\text { MPAl from baseline to T4 }(M=101.33): t(11)=3.94, p<.05 \text {, } \\
\text { Hedges' } g=2.19 \text {. } \\
\text { ACQ from baseline }(M=74.86) \text { to T2 }(M=94): t(11)=3.04 \text {, } \\
p<.05, \text { Hedges' } g=1.64, \text { ACQ from baseline to T3 }(M= \\
\text { 95.33): } t(11)=3.12, p<.05, \text { Hedges' } g=1.70, \text { ACQ from } \\
\text { baseline to T4 }(M=95.67): t(11)=3.15, p<.05, \text { Hedges' } g= \\
\text { 1.72. }\end{array}$ & 11 \\
\hline $\begin{array}{l}\text { Khalsa \& Cope, } \\
2006\end{array}$ & $\begin{array}{l}\text { Musicians } \\
\text { taking part at a } \\
\text { training } \\
\text { program, age: } \\
21-30 \\
\text { years, } 50 \% \\
\text { women, } 50 \% \\
\text { men, }\end{array}$ & $\begin{array}{l}N=18 \\
\text { Group 1: } N=10 \\
\text { group 2: } N=8\end{array}$ & $\begin{array}{l}\text { Intervention study (not } \\
\text { randomized), pre }=\mathrm{T} 1 \\
\text { post }=\mathrm{T} 2\end{array}$ & $\begin{array}{l}\text { MPA investigated with: PAQ (subscales: } \\
\text { solo, practice and group) } \\
\text { Treatment: group 1: } 8 \text { weeks of yoga } \\
\text { lifestyle intervention group, group 2: no } \\
\text { practice control group }\end{array}$ & $\begin{array}{l}\text { PAQ solo, (but not practice and group scores) changed } \\
\text { significantly for group } 1(p=.05) \text {, but not for the group2 from } \\
\text { T1 to T2. }\end{array}$ & -4 \\
\hline $\begin{array}{l}\text { Khalsa et al., } \\
2009\end{array}$ & $\begin{array}{l}\text { Musicians } \\
\text { taking part at a } \\
\text { training } \\
\text { program, age: } \\
\text { group } 1: M= \\
24.5, S D=2.4, \\
\text { group } 2: M= \\
25.4, S D=3.9, \\
\text { group } 3: M= \\
24.0, S D=1.6,\end{array}$ & $\begin{array}{l}N=45 \\
\text { Group 1: } N=15, \\
\text { group 2: } N=15, \\
\text { group 3: } N=15\end{array}$ & $\begin{array}{l}\text { Intervention study (only } \\
\text { yoga interventions } \\
\text { were randomized for } \\
\text { musicians being } \\
\text { interested in yoga), pre } \\
=\mathrm{T} 1, \text { post }=\mathrm{T} 2,10- \\
\text { month follow-up }=\mathrm{T} 3\end{array}$ & $\begin{array}{l}\text { MPA investigated with: PAQ } \\
\text { Risk factor: gender } \\
\text { Treatment: group 1: } 8 \text { weeks of yoga } \\
\text { lifestyle intervention, group 2: } 8 \text { weeks of } \\
\text { yoga only, group3: control group without } \\
\text { intervention, }\end{array}$ & $\begin{array}{l}\text { Risk factor: Women reported higher PAQ scores on all } \\
\text { scales (about 2.4-8.4 points higher), but not statistically } \\
\text { different to males. } \\
\text { Treatment: Group 2: T1 to T2 PAQ practice (difference } \\
\text { value: }=-6.47, \text { SEM }=+/-2.30 \text { ), solo (difference value: }-5.87 \text {, } \\
\text { SEM }=+/-2.69 \text { ) and group performance (difference value: - } \\
5.23, S E M=+/-2.38 \text { ) scores changed significantly (all } p< \\
.05 \text { ) and remained significant from T1 to T3 for PAQ solo and } \\
\text { group. } \\
\text { Group 1: T1 to T2 PAQ group (difference value: }-5.23, S E M \\
=+/-2.37 \text { ) and solo (difference value: }-5.29, S E M=+/-2.45 \text { ) }\end{array}$ & -2 \\
\hline
\end{tabular}




\begin{tabular}{|c|c|c|c|c|c|c|}
\hline & $\begin{array}{l}55.56 \% \text { female, } \\
44.44 \% \\
\text { male }\end{array}$ & & & & $\begin{array}{l}\text { reduced significantly (both } p<.05 \text { ). Practice Scores did not } \\
\text { change (difference value: }-4.70, S E M=+/-2.52 \text { ). Scores } \\
\text { from T1 to T3 did not change significantly. } \\
\text { Group 3: all scores remained unchanged from T1 to T2: } \\
\text { practice (difference value: }-1.60, S E M=+/-1.74 \text { ), group } \\
\text { performance (difference value: }-0.40, S E M=+/-2.12 \text { ) and } \\
\text { solo performance (difference value: }-0.57, S E M=+/-2.15 \text { ). } \\
\text { No change from T1 to T3 } \\
\text { Between group comparisons: no significant differences at } \\
\text { any time between the PAQ scores of group } 1,2 \text { and } 3 \text {. }\end{array}$ & \\
\hline Kim, 2005 & $\begin{array}{l}\text { College } \\
\text { students, piano, } \\
\text { age: } M=25 \\
S D=2.42 \text {, all } \\
\text { female with } \\
\text { MPA }\end{array}$ & $N=6$ & $\begin{array}{l}\text { Intervention study (no } \\
\text { control group), pre = } \\
\mathrm{T} 1 \text {, post }=\mathrm{T} 2\end{array}$ & $\begin{array}{l}\text { MPA investigated with: visual analogue } \\
\text { scale of MPA (VAS: } 1=\text { least anxious, } \\
10=\text { most anxious), STAI-S, STAI-T, } \\
\text { PARQ } \\
\text { Treatment: Music therapy (with } \\
\text { improvisation and desensitization), } 30 \\
\text { minutes per week, for } 6 \text { weeks }\end{array}$ & $\begin{array}{l}\text { Significant changes from T1 to T2: } \\
\text { VAS: } \mathrm{T} 1: M=7.33, S D=2.16, \mathrm{~T} 2: M=5.33, S D=2.07, \\
\text { p(T1, T2) }=.02, \\
\text { STAI-S: } \mathrm{T} 1: M=61.17, S D=15.08, \mathrm{~T} 2: M=50.67, S D= \\
\text { 14.11, } p(\mathrm{~T} 1, \mathrm{~T} 2)=.03 . \\
\text { No significant changes for: } \\
\text { PARQ: T1: } M=78.50, S D=15.20, \mathrm{~T} 2: M=74.67, S D= \\
\text { 12.18, } p(\mathrm{~T} 1, \mathrm{~T} 2)=.50, \\
\text { STAI-T: } \mathrm{T} 1: M=36.67, S D=8.91, \mathrm{~T} 2: M=36.17, S D=7.83 \\
\text { p(T1,T2) }=.46 .\end{array}$ & 4 \\
\hline Kim, 2008 & $\begin{array}{l}\text { Student } \\
\text { pianists, age: } M \\
=20, \text { all female }\end{array}$ & $\begin{array}{l}N=30, N(\text { group } \\
1)=15, N(\text { group } \\
2)=15\end{array}$ & $\begin{array}{l}\text { Intervention study } \\
(\mathrm{RCT}), \text { pre }=\mathrm{T} 1, \text { post }= \\
\mathrm{T} 2\end{array}$ & $\begin{array}{l}\text { MPA investigated with: STAI-S, MPAQ, } \\
\text { visual analogue scale of MPA from } 0 \\
\text { (low anxiety) to } 15 \text { (high anxiety) } \\
\text { Treatment: } 6 \text { weekly sessions, group 1: } \\
\text { Music therapy improvisation and } \\
\text { desensitization protocol (MTIDP), group } \\
\text { 2: music-assisted progressive muscle } \\
\text { relaxation and imagery }\end{array}$ & 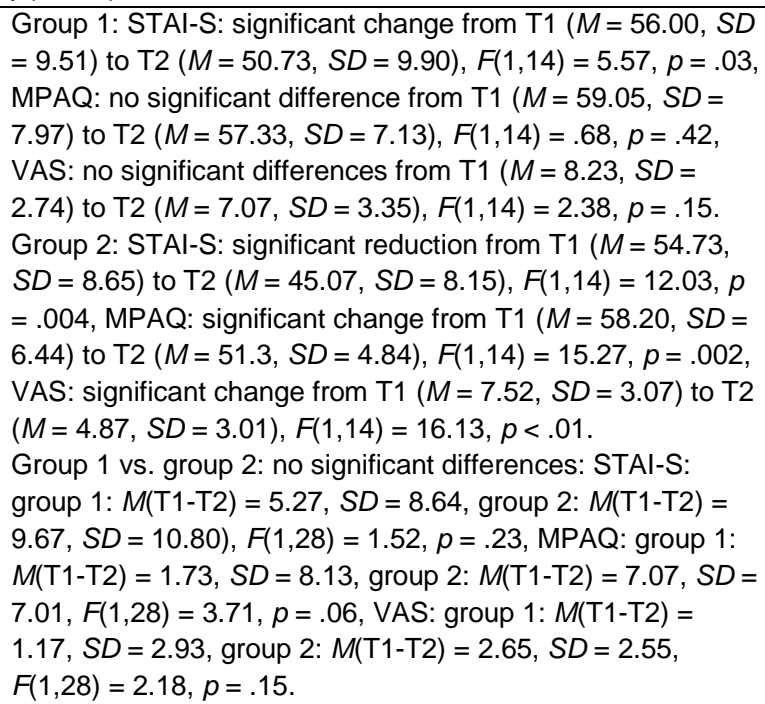 & 4 \\
\hline $\begin{array}{l}\text { Montello et al., } \\
1990\end{array}$ & $\begin{array}{l}\text { Experiment } 1: \\
\text { Freelance } \\
\text { musicians, age: } \\
M=28, \text { range: } \\
18-48 \text { years, }\end{array}$ & $\begin{array}{l}N=17, N(\text { group } \\
\text { 1) }=7, N(\text { group } \\
\text { 2) }=10\end{array}$ & $\begin{array}{l}\text { Intervention study } \\
(\mathrm{RCT}), \text { pre }=\mathrm{T} 1, \text { post }= \\
\mathrm{T} 2\end{array}$ & $\begin{array}{l}\text { MPA investigated with: STAI-T, PRCP } \\
\text { Treatment: group 1: } 12 \text { week music } \\
\text { group therapy (one session/week), group } \\
\text { 2: waitlist }\end{array}$ & $\begin{array}{l}\text { STAl-T: group 1: } M(\mathrm{~T} 1)=47.43, S E=3.75, M(\mathrm{~T} 2)=41.43, \\
S E=4.10, \text { group } 2: M(\mathrm{~T} 1)=47.70, S E=3.21, M(\mathrm{~T} 2)= \\
48.30, S E=3.04 \\
F(1,13)=7.4, p<.013\end{array}$ & -4 \\
\hline
\end{tabular}




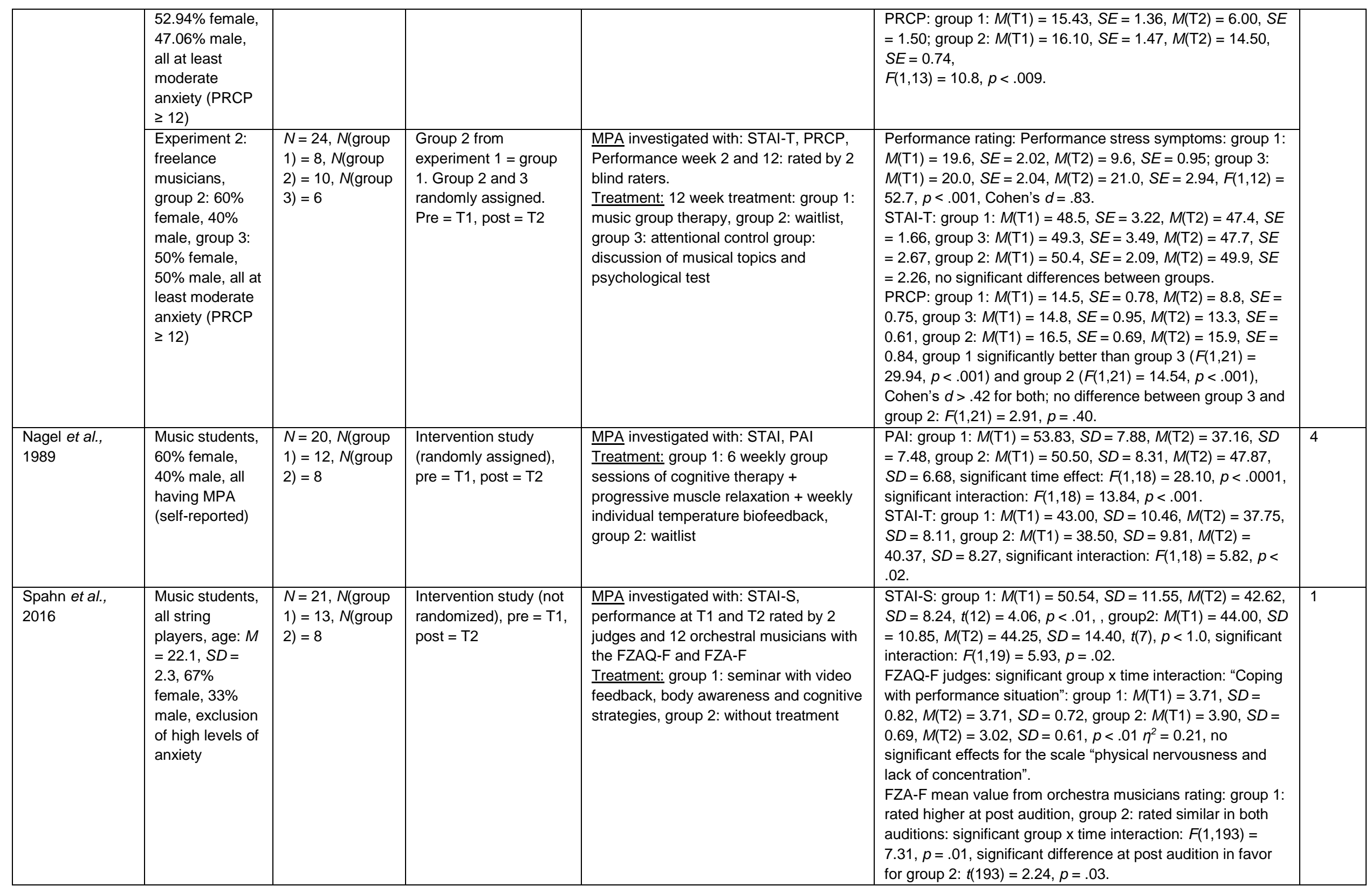




\begin{tabular}{|c|c|c|c|c|c|c|}
\hline Stanton, 1994 & $\begin{array}{l}\text { Music students } \\
\text { with MPA } \\
\text { (detected by } \\
\text { their lecturers) }\end{array}$ & $\begin{array}{l}N=40, N(\text { group } \\
1)=20, N(\text { group } \\
2)=20\end{array}$ & $\begin{array}{l}\text { Intervention study } \\
\text { (paired with PAl score } \\
\text { and randomly } \\
\text { assigned), pre = T1, } \\
\text { post = T2, 6-month } \\
\text { follow-up = T3 }\end{array}$ & $\begin{array}{l}\text { MPA investigated with: PAl } \\
\text { Treatment: group 1: } 2 \text { weekly } \\
\text { hypnotherapeutical sessions, group 2: } \\
\text { weekly sessions with discussion about } \\
\text { their courses }\end{array}$ & $\begin{array}{l}\text { PAl: group 1: } M(\mathrm{~T} 1)=69.7, S D=8.5, M(\mathrm{~T} 2)=59.1, S D= \\
\text { 8.3, } M(\mathrm{~T} 3)=42.8, S D=8.2 \text {, from T1 to T2: } t(19)=4.38, p< \\
.01, \text { from T1 or T2 (not reported which time point was used) } \\
\text { to T3: } t(19)=6.30, p<.01 \text {. group } 2: M(\mathrm{~T} 1)=64.8, S D=7.7 \text {, } \\
M(\mathrm{~T} 2)=61.8, S D=8.7, M(\mathrm{~T} 3)=58.8, S D=8.1, \text { no } \\
\text { significant difference from T1 to T2, but from T1 or T2 (not } \\
\text { reported) to T3 } t(19)=.42, p<.05 . \\
\text { T3: group } 1 \text { lower PAl than group2: } t(19)=5.63, p<.01 \text {. }\end{array}$ & 2 \\
\hline $\begin{array}{l}\text { Stern et al., } \\
2012\end{array}$ & $\begin{array}{l}\text { Music students, } \\
\text { age: } M=21.7 \\
S D=3.1 \\
87.5 \% \text { female } \\
12.5 \% \text { male }\end{array}$ & $N=24$ & $\begin{array}{l}\text { Intervention study (no } \\
\text { control group), } \\
\text { Pre = T1, Post = T2 }\end{array}$ & $\begin{array}{l}\text { MPA investigated with: PAQ (subscales: } \\
\text { solo, group and practice scores), K- } \\
\text { MPAI, STAI-T } \\
\text { Treatment: } 14 \text { classes of yoga, twice a } \\
\text { week (each } 1 \text { hour) and home practice } 4 \\
\text { days per week }\end{array}$ & 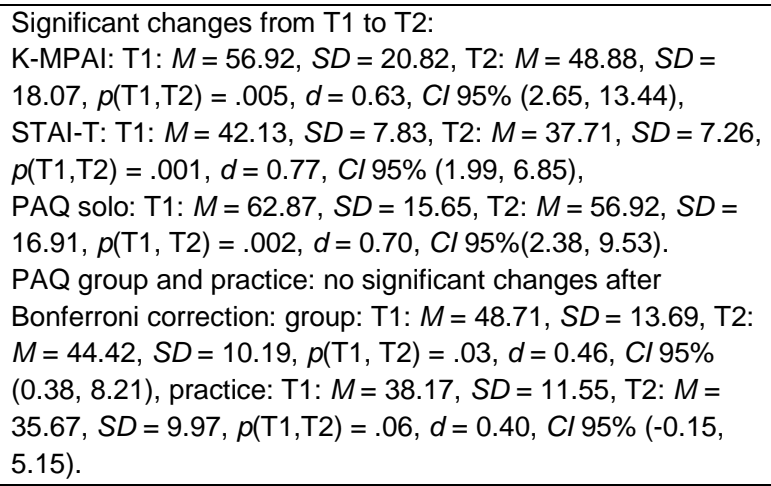 & 0 \\
\hline $\begin{array}{l}\text { Sweeney \& } \\
\text { Horan, } 1982\end{array}$ & $\begin{array}{l}\text { Music students } \\
\text { of piano class, } \\
\text { screened for } \\
\text { MPA, } 48.98 \% \\
\text { female, } 51.02 \% \\
\text { male }\end{array}$ & $\begin{array}{l}N=49, N=9-10 \\
\text { persons per } \\
\text { group }\end{array}$ & $\begin{array}{l}\text { Intervention study } \\
\text { (randomized with } \\
\text { respect to MPA } \\
\text { scores), pre = T1, post } \\
=\mathrm{T} 2\end{array}$ & $\begin{array}{l}\text { MPA investigated with: Adaptation of } \\
\text { AATS, AD, video and audiotaped public } \\
\text { recital at T1 and T2: rated for musical } \\
\text { performance competence (MPC = } \\
\text { number of errors) and behavioral index } \\
\text { of anxiety (BIA = number of MPA } \\
\text { symptoms seen on video), } \\
\text { Treatment: six weekly sessions of group } \\
\text { therapy group 1: cue-controlled } \\
\text { relaxation, group 2: cognitive } \\
\text { restructuring, group 3: cue-controlled } \\
\text { relaxation + cognitive restructuring, } \\
\text { group 4: standard treatment control } \\
\text { group = musical analysis training, group } \\
5: \text { waitlist }\end{array}$ & $\begin{array}{l}\text { Group 1: significant results for AATS, debilitating subscale: } \\
M(\mathrm{~T} 1)=30.55, S D=5.24, M(\mathrm{~T} 2)=27.55, S D=6.57, F(1,31 \\
)=4.02, p<.05, \mathrm{MPC}: M(\mathrm{~T} 1)=19.88, S D=10.55, M(\mathrm{~T} 2)= \\
7.11, S D=5.18, F(1,31)=15.90, p<.001 \text { and } \mathrm{AD}: M(\mathrm{~T} 1)= \\
\text { 10.11, } S D=4.28, M(\mathrm{~T} 2)=4.66, S D=3.60, F(1,31)=9.47, p \\
<.004 . \\
\text { Group 2: significant results for BIA: } M(\mathrm{~T} 1)=38.94, S D= \\
\text { 13.24, } M(\mathrm{~T} 2)=20.21, S D=14.77, F(1,31)=10.08, p<.003 \text {. } \\
\text { Group 3: significant results for AATS, debilitating subscale: } \\
M(\mathrm{~T} 1)=33.11, S D=6.60, M(\mathrm{~T} 2)=24.88, S D=6.11, \mathrm{AD}: \\
M(\mathrm{~T} 1)=11.55, S D=3.90, M(\mathrm{~T} 2)=5.22, S D=3.63, \mathrm{BIA}: \\
M(\mathrm{~T} 1)=43.07, S D=20.59, M(\mathrm{~T} 2)=17.65, S D=27.10, \\
\text { MPC: } M(\mathrm{~T} 1)=19.22 S D=9.12, M(\mathrm{~T} 2)=10.44, S D=3.61, \\
\text { no test statistics reported. } \\
\text { Group } 4 \text { and } 5: \text { no significant changes from T1 to T2, and no } \\
\text { differences between the } 2 \text { groups, no test statistics reported. }\end{array}$ & 4 \\
\hline $\begin{array}{l}\text { Valentine et al., } \\
1995\end{array}$ & $\begin{array}{l}\text { Music students, } \\
\text { age: } M=20.9 \\
S D=2.4,84 \% \\
\text { female, } 16 \% \\
\text { male }\end{array}$ & $\begin{array}{l}N=25, N(\text { group } \\
1)=12, N(\text { group } \\
2)=13\end{array}$ & $\begin{array}{l}\text { Intervention study } \\
\text { (randomly assigned), } \\
\text { pre }=\mathrm{T} 1 \text {, post }=\mathrm{T} 2\end{array}$ & $\begin{array}{l}\text { MPA investigated with: PAI, } 4 \\
\text { performance situations: } \\
\text { high stress (T1: one staff member, T2: } \\
\text { public recital) and low stress (T1 and T2: } \\
\text { in front of their class), judgement of } \\
\text { music quality and technical quality by } 2 \\
\text { independent experts, NMAC (anxiety), } \\
\text { MPASS }\end{array}$ & $\begin{array}{l}\text { Risk factor: significant relationship between neuroticism and } \\
\text { PAI }(r=.59, p=.003) \\
\text { Rated music quality: significant interaction time by group low } \\
\text { stress (T1 and T2): } F(1,20)=3.48, p=.04 \text {, group } 1 \text { showed } \\
\text { improvement, group } 2 \text { declined. } \\
\text { Rated technical quality: significant interaction time by group } \\
\text { low stress (T1 and T2): } t(1)=2.41, p=.03 \text {, group } 1 \text { showed } \\
\text { improvement, group } 2 \text { declined. }\end{array}$ & 2 \\
\hline
\end{tabular}




\begin{tabular}{|c|c|c|c|c|c|c|}
\hline & & & & $\begin{array}{l}\text { Risk factor: neuroticism (Eysenck \& } \\
\text { Eysenck) } \\
\text { Treatment: group 1: } 15 \text { lessons of } \\
\text { Alexander technique, group 2: no } \\
\text { treatment }\end{array}$ & $\begin{array}{l}\text { NMAC (anxiety): time effect from T1 to T2 } F(1,19)=6.39, p \\
=.02, \text { group } 1 \text { significantly more improvement than group } 2 \text { : } \\
t(1)=1.83, p=.04 \text {. } \\
\text { MPASS: Interaction time by group T1 to T2 (low stress): } \\
F(1,19)=4.25, p=.05 \text {, group } 1 \text { increased, group } 2 \text { declined. }\end{array}$ & \\
\hline $\begin{array}{l}\text { Wells et al., } \\
2012\end{array}$ & $\begin{array}{l}\text { Musicians, age: } \\
M=30.4, S D= \\
11.98,52.17 \% \\
\text { Female, } \\
47.83 \% \text { male, } 9 \\
\text { of them having } \\
\text { a history of } \\
\text { mental illness }\end{array}$ & $\begin{array}{l}N=46 \\
N(\text { group } 1)=14, \\
N(\text { group } 2)=15 \\
N(\text { group } 3)=15 \\
\text { dropouts: } 2\end{array}$ & $\begin{array}{l}\text { Intervention study } \\
(\mathrm{RCT}), \text { pre }=\mathrm{T} 1 \text {, post }= \\
\text { T2 }\end{array}$ & $\begin{array}{l}\text { Anxiety investigated with: heart rate } \\
\text { variability (HRV): high frequency (HF) } \\
\text { HRV, LF/HF ratio, STAI-S, all measured } \\
\text { at T1 and T2 before a performance and } \\
\text { at a resting situation } \\
\text { Treatment: group 1: } 30 \text { minutes slow } \\
\text { breathing with low frequency (LF) HRV } \\
\text { biofeedback, group 2: } \\
\text { Breathing control group: slow breathing } \\
\text { without HRV biofeedback, group 3: } \\
\text { control group: reading }\end{array}$ & $\begin{array}{l}\text { HF HRV and LF/HF ratio: no significant main effects for time } \\
\text { or group or interactions. Taken group } 1 \text { and } 2 \text { together } \\
\text { significant improvement of HF HRV }(\eta=0.122) \text { and LF/HF } \\
\text { ratio }(\eta=0.116) \text { compared to group } 3 \text { during anxious } \\
\text { anticipation at T2. } \\
\text { STAI-S: no significant main effects for time or group or } \\
\text { interactions. Looking only at participants with high resting } \\
\text { STAI-S scores, participants of group } 1 \text { and } 2 \text { displayed } \\
\text { significant greater reductions in STAI-S than group } 3 \\
\text { participants }(U=21.5, p=.05, r=0.379) \text {. }\end{array}$ & 6 \\
\hline
\end{tabular}

$N=$ number of participants, $M=$ mean, $S D=$ standard deviation, $S E=$ standard error, outcome and results = prevalence, risk factor or treatment of $M P A$, rating $=$ quality rating of study, $M P A=$ mus

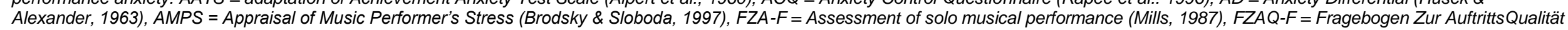
- Fremdeinschätzung: (Spahn, et al., 2013), KAB = "Kurzfragebogen zur aktuellen Belastung" (Müller \& Basler, 1993), K-MPAI = Kenny Music Performance Anxiety Inventory (Kenny, 2009), MPAQ = The Music Performance Anxiety Questionnaire (Lehrer et al., 1993), MPASS = Music performance anxiety self-statement scale (Craske et al., 1988): positive outlook and task-focused attention, MPQ = Music Performance Quality Rating Form (Educational Testing Service, 1998), MPSS = Music Performance Stress Survey (Brodsky \& Sloboda, 1997), NMAC = Nowlis mood adjective checklist (Nowlis,

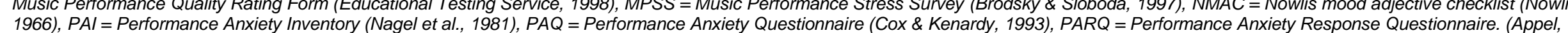
1976), POA = "Podiumsangst" (Schröder \& Liebelt, 1999), PRCP = Personal Report of Confidence as a Performer (Appel, 1976), STAI-S= State Anxiety Inventory, STAI-T= Trait Anxiety Inventory (Spielberger et al., 1982). 


\section{Table 3: Case studies}

\begin{tabular}{|c|c|c|c|c|c|}
\hline Study & Population & $\mathrm{N}$ & Design & Outcome & Results \\
\hline $\begin{array}{l}\text { Abilgaard, } \\
2007\end{array}$ & $\begin{array}{l}\text { Brass player, } 51 \text { years, male, } \\
\text { MPA and alcohol dependency }\end{array}$ & $N=1$ & & $\begin{array}{l}\text { MPA investigated with: self-report } \\
\text { Treatment: alcohol detoxification in hospital, Alexander } \\
\text { technique, stress management with mental training, } \\
\text { reactivation of hobbies }\end{array}$ & $\begin{array}{l}\text { Patient is not suffering from MPA after } \\
\text { intervention. He had a relapse once and is now } \\
\text { abstinent since } 14 \text { month. }\end{array}$ \\
\hline $\begin{array}{l}\text { Juncos \& } \\
\text { Markman, } \\
2016\end{array}$ & $\begin{array}{l}\text { Violinist, sophomore Music } \\
\text { Education major, female, MPA }\end{array}$ & $N=1$ & $\begin{array}{l}\mathrm{T} 1=\text { pre, } \mathrm{T} 2=\text { post, } \\
\mathrm{T} 3=1 \text {-month follow- } \\
\text { up }\end{array}$ & $\begin{array}{l}\text { MPA investigated with: K-MPAI, PAI, ACQ and rated } \\
\text { performance by judge T1 and T2 with MPQ } \\
\frac{\text { Treatment: } 10 \text { sessions of Acceptance and Commitment }}{\text { Therapy }}\end{array}$ & 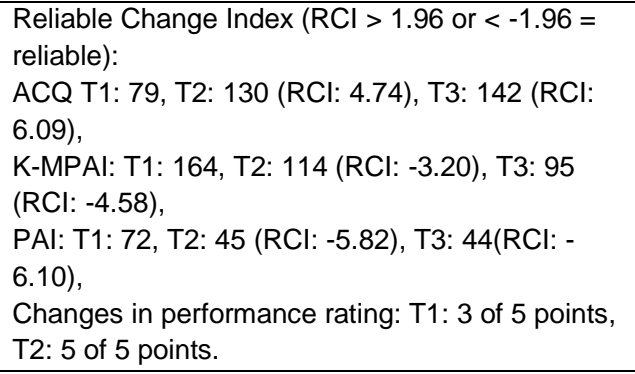 \\
\hline $\begin{array}{l}\text { Kenny et al., } \\
2016\end{array}$ & $\begin{array}{l}\text { Orchestral musician, string } \\
\text { player, age: } 55 \text {, male, MPA }\end{array}$ & $N=1$ & & $\begin{array}{l}\text { Treatment: Intensive Short-Term Dynamic } \\
\text { Psychotherapy } 10 \text { sessions }\end{array}$ & Authors report that the Patient resolved MPA. \\
\hline $\begin{array}{l}\text { Lazarus \& } \\
\text { Abramovitz, } \\
2004\end{array}$ & Violinist, male, MPA & $N=1$ & & $\begin{array}{l}\text { MPA investigated with: self-report } \\
\text { Treatment: } 3 \text { month of individual systematic } \\
\text { desensitization therapy with } 20 \text { sessions }\end{array}$ & $\begin{array}{l}\text { After 6-7 week follow-up: completely cured (self- } \\
\text { report). }\end{array}$ \\
\hline Moreno, 1946 & $\begin{array}{l}\text { Violinist, age: } 45 \text { years, male } \\
\text { MPA }\end{array}$ & $N=1$ & & Treatment: Psychodramatic treatment & Author reports less MPA at the end of treatment. \\
\hline $\begin{array}{l}\text { Norton et al., } \\
1978\end{array}$ & $\begin{array}{l}\text { Piano player, age: } 20 \text { years, } \\
\text { female, MPA }\end{array}$ & $N=1$ & & $\begin{array}{l}\text { MPA investigated with: piano concert self-report } \\
\text { Treatment: } 15 \text { sessions of individual systematic } \\
\text { desensitization therapy and Meichenbaums verbal self- } \\
\text { directed positive statements training. }\end{array}$ & $\begin{array}{l}\text { Patient reported no more anxiety during piano } \\
\text { concerts after therapy. }\end{array}$ \\
\hline Rider, 1987 & $\begin{array}{l}\text { Cellist, age: } 34 \text { years, female, } \\
\text { MPA }\end{array}$ & $N=1$ & & $\begin{array}{l}\text { MPA investigated with: self-report } \\
\text { Treatment: } 8 \text { sessions of individual music } \\
\text { psychotherapy, biofeedback, systematic desensitization, } \\
\text { and cognitive restructuring }\end{array}$ & $\begin{array}{l}\text { Patient reported more self-confidence, satisfaction } \\
\text { from playing and improved playing at follow-up. }\end{array}$ \\
\hline $\begin{array}{l}\text { Safirstein, } \\
1962\end{array}$ & $\begin{array}{l}\text { Piano player, viola and cello, } \\
\text { age: } 34 \text { years, female, MPA }\end{array}$ & $N=1$ & & $\begin{array}{l}\text { MPA investigated with: self-report } \\
\text { Treatment: } 200 \text { hours of psychoanalysis }\end{array}$ & $\begin{array}{l}\text { At the end of therapy patient reported having no } \\
\text { more anxiety. }\end{array}$ \\
\hline Salmon, 1992 & $\begin{array}{l}\text { Oboist, age: } 47 \text { years, female, } \\
\text { MPA }\end{array}$ & $N=1$ & $\mathrm{~T} 1=$ pre, $\mathrm{T} 2=$ post & $\begin{array}{l}\text { MPA investigated with BAI, ASI } \\
\text { Treatment: } 8 \text { sessions of CBT }\end{array}$ & $\begin{array}{l}\text { BAI T1: } 23, \text { T2: } 6 \\
\text { ASI T1: } 27, \text { T2: } 13\end{array}$ \\
\hline Stanton, 1993 & $\begin{array}{l}\text { Violin player, age: } 38 \text { years, } \\
\text { piano music student, age: } 22 \\
\text { years, guitarist, age: } 27 \text { years, } \\
\text { all having MPA }\end{array}$ & $N=3$ & $\begin{array}{l}\text { T1 }=\text { pre }, \text { T2 }=\text { post } \\
\text { T3 }=6 \text {-month follow- } \\
\text { up }\end{array}$ & $\begin{array}{l}\text { MPA investigated with: PAl } \\
\text { Treatment: } 2 \text { sessions of hypnotherapy, success } \\
\text { imagery and rational emotive therapy }\end{array}$ & $\begin{array}{l}\text { Violin player: T1: } 67.8, \text { T2 43.3, T3: } 36.7, \\
\text { Music student: T1: 64.9, T2: 48.3, T3: 39.45, } \\
\text { Guitarist: T1: } 74.3, \text { T2: 61.4, T3: 41.2. }\end{array}$ \\
\hline
\end{tabular}

$N=$ number of participants, $M=$ mean, $S D=$ standard deviation, Outcome and results = prevalence, risk factor or treatment of MPA, rating = quality rating of study, MPA = music performance anxiety $A C Q=$ Anxiety Control Questionnaire (Rapee et al., 1996), ASI = Anxiety Sensitivity Index (Reiss et al. 1986), BAI = Beck Anxiety Inventory (Beck \& Steer, 1993), K-MPAI = Kenny Music Performance Anxiety Inventory (Kenny, 2009), MPQ = Music Performance Quality Rating Form (Craske et al., 1988), PAI = Performance Anxiety Inventory (Nagel et al., 1981). 


\subsection{Prevalence and incidence}

Prevalence of MPA was between 16.5-60\% (Fishbein et al., 1988, Krawehl and Altenmüller, 2000, Modeiros Barbar et al., 2014, Middlestadt, 1990, Sousa et al., 2016, van Kemenade et al., 1995, Wesner et al., 1990). About one third of the examined musicians indicated MPA to be a severe problem (24\% (Fishbein et al., 1988, Middlestadt, 1990), 38\% (Krawehl and Altenmüller, 2000), 39\% (Modeiros Barbar et al., 2014), 21.5\% (Sousa et al., 2016), $16.5 \%$ (Wesner et al., 1990) and 58.7\% (van Kemenade et al., 1995)), whilst about $60 \%$ of them at least once experienced some kind of MPA in their career (Krawehl and Altenmüller, 2000). MPA was mostly assessed by self-reports of patients and not by professionals according to ICD or DSM criteria. For incidence no studies were found.

\subsection{Risk Factors}

The majority of studies reported about different gender distribution. Women were found to be more frequently affected by MPA than men or displayed higher scores on questionnaires addressing MPA (Fishbein et al., 1988, Hildebrandt et al., 2012, Kenny et al., 2014, Middlestadt, 1990, Wesner et al., 1990). However, some studies could not find differences between men and women (Kenny et al., 2004, Khalsa et al., 2009, van Kemenade et al., 1995). Regarding age, younger musicians seem to be more affected from MPA than older musicians (Fishbein et al., 1988, Kenny et al., 2014, Middlestadt, 1990, Steptoe and Fidler, 1987). With an age older than about 45-50, there is a tendency to less MPA. Two studies found no relationship between age and MPA (van Kemenade et al., 1995, Wesner et al., 1990). In three studies there was a positive relationship between neuroticism and MPA (Hodapp et al., 2009, Steptoe and Fidler, 1987, Valentine et al., 1995) and one study found a negative relationship between selfefficacy and MPA (Hodapp et al., 2009). Another study found that symphonic orchestra musicians suffer most from MPA (van Kemenade et al., 1995). Regarding type of instrument, one study found brass players to be most affected by MPA (Fishbein et al., 1988, Middlestadt, 1990), but another study could not find any differences between instruments (Kenny et al., 2014). 


\subsection{Treatment}

Different treatments for MPA have been investigated. Most often research was conducted for $\mathrm{CBT}$, relaxation, exercise and beta blockers. Not all studies reported how and if diagnoses of MPA were determined and in four studies all participants were unaffected of mental illness (and therefore also free from MPA in sense of ICD or DSM). Results of those four studies are reported separately. To measure MPA different questionnaires were used (for example: Kenny Music Performance Anxiety Inventory (K-MPAI) (Kenny, 2009), Performance Anxiety Inventory (PAI) (Nagel et al., 1981) or State Anxiety Inventory (STAI-S) (Spielberger et al., 1982)). Furthermore, self-ratings of anxiety or performance quality (rated by a jury or self-rating of musicians) were used as measures of MPA. There were different types of control groups ranging from waiting list, to non-active or active control groups. Some studies are considered several times as they compared different interventions with each other.

\subsubsection{Psychological counselling}

Psychological counselling in general was rated to be helpful in about $60-62 \%$ by patients in three cross-sectional studies (Fishbein et al., 1988, Kenny et al., 2014, Middlestadt, 1990).

\subsubsection{CBT}

Ten studies investigated CBT. Different CBT techniques were examined and all showed positive effects on MPA. There were two intervention studies without control group: Brodksy and Sloboda (1997) found a significant reduction of MPA after a CBT intervention alone or plus relaxation with or without music. Juncos, et al. (2017) researched 12 sessions of acceptance and commitment therapy (ACT). The authors found a significant reduction of MPA. Ratings of performance quality did not change before and after therapy.

In three studies CBT was compared to a non-active or a waiting list control group. One of those studies investigated six sessions of virtual reality exposure training (Bissonnette et al., 2015). MPA reduced significantly after therapy and the quality of performances after therapy improved significantly compared to before and compared to waiting list control group performances. Another study comprised six group sessions of cognitive therapy, PMR and weekly individual temperature biofeedback (Nagel et 
al., 1989). MPA reduced significantly after the intervention and the CBT group had less MPA than the control group. Sweeney and Horan (1982) examined six group sessions of cognitive restructuring with or without cue controlled relaxation and found that anxiety symptoms seen on a videotape of a performance significantly decreased after therapy compared to before. For cognitive restructuring with cue-controlled relaxation MPA decreased significantly. An active control group (musical analysis training) and a waiting list control did not change in their MPA level in the same time (Sweeney and Horan, 1982).

There were five case reports investigating systematic desensitization, verbal selfdirected positive statements training, cognitive restructuring and ACT (Juncos and Markman, 2016, Lazarus and Abramovitz, 2004, Norton et al., 1978, Rider, 1987, Salmon, 1992). All patients reported that MPA improved or was cured after therapy.

\subsubsection{Psychoanalytic and psychodynamic therapy}

There were two case reports investigating psychoanalytic and psychodynamic therapy in MPA: Safirstein (1962) reported that after 200 hours of psychoanalysis the patient had no more anxiety and Kenny, Arthey and Abbass (2016) investigated 10 sessions of intensive short-term dynamic psychotherapy and found a positive effect on MPA.

\subsubsection{Music Therapy}

Music therapy improvisation and desensitization was investigated in four trials (Kim, 2005, 2008, Montello et al., 1990). All showed a significant reduction of MPA after the intervention. In two studies music therapy resulted in significantly lower MPA compared to a waiting list condition and an active control group (discussion of musical topics) (both Montello et al. (1990)). One study compared music therapy to PMR and imagery. Both interventions equally reduced MPA (Kim, 2008).

\subsubsection{Beta blockers}

As a pharmacological treatment approach of MPA, the effect of beta blockers was quite intensively studied. In one cross-sectional study beta blockers were rated to be helpful by $93 \%$ of musicians (Kenny et al., 2014). One randomised controlled trial tested $20 \mathrm{mg}$ nadolol versus placebo (Gates and Montalbo, 1987). There was no significant difference in anxiety before performances and in quality ratings of performances between both groups. Another randomised controlled trial tested 0, 20, 40 and $80 \mathrm{mg}$ 
of nadolol compared to placebo. The active groups did not differ regarding quality of performance and self-rated anxiety levels from placebo but heart rate was significantly lower in all nadolol conditions. Comparing only nadolol groups to each other, $20 \mathrm{mg}$ of nadolol revealed best effects on MPA (Gates et al., 1985). An intervention study compared $25 \mathrm{mg}$ of propranolol (administered once 1-1.5 hours before performance) and a few weeks of PMR (Hinz, 2005). Propranolol reduced heart rate more significantly than PMR. There was no significant difference in MPA between the two groups, but for the PMR group there was a significantly lower disease-related burden after the performance.

\subsubsection{Exercise}

Within a cross-sectional study, aerobic exercise was found to be effective against MPA in $70 \%$ of musicians and yoga was rated as a helpful intervention in $58 \%$ (Fishbein et al., 1988, Middlestadt, 1990). Two intervention studies found significant reductions of MPA in questionnaires after yoga interventions (8 weeks/14 classes) (Khalsa and Cope, 2006, Stern, 2012). One intervention study compared a yoga lifestyle intervention, yoga only and a no treatment control group. Both yoga groups lead to significant improvements in MPA. But at no time point yoga groups differed significantly to the no treatment control group regarding MPA (Khalsa et al., 2009).

\subsubsection{Hypnotherapeutical interventions}

In cross-sectional studies hypnosis was rated to be helpful against MPA by $60 \%$ to $76 \%$ of musicians (Fishbein et al., 1988, Kenny et al., 2014, Middlestadt, 1990). Stanton (1994) investigated hypnotherapy compared to an active control group (talking about courses). He found a significant reduction in MPA (measured by a questionnaire) after the intervention and at follow-up. At follow-up hypnotherapy resulted in a significantly lower MPA than the control group intervention.

\subsubsection{Relaxation techniques}

Relaxation techniques were rated to be helpful by $12 \%$ of musicians and deep breathing was rated to be helpful by 41 to $78 \%$ of the musicians in cross-sectional studies (Hiner et al., 1987, Kenny et al., 2014).

Two studies examined PMR: MPA severity decreased significantly after musicassisted PMR plus imagery and PMR was as effective as music therapy (Kim, 2008), 
and as already reported (see beta blockers) PMR lead to a lower burden directly after performance compared to beta blockers. Chang, Midlarsky and Lin (2003) found a significant reduction of MPA after a meditation intervention but no significant difference to a waiting list control group in MPA after the intervention.

Six group sessions of cue-controlled relaxation resulted in a significant reduction of MPA, whilst an active (musical analysis training) and waiting list control group did not change within the same period of time (Sweeney and Horan, 1982).

\subsubsection{Mixed interventions}

Two case reports investigated several interventions: Stanton (1993) found decreased MPA in three patients after two sessions of hypnotherapy, success imagery and rational emotive therapy. Abilgaard (2007) investigated Alexander technique, stress management with mental training and reactivation of hobbies in a patient with MPA and alcohol abuse (after alcohol detoxification). After the treatment the patient was not suffering from MPA anymore.

\subsubsection{Other interventions}

In cross-sectional studies the following interventions were rated to be helpful against MPA: Alexander technique by $47 \%$ (Fishbein et al., 1988, Middlestadt, 1990), focusing on performance by $69 \%$ (Hiner et al., 1987), mock performance practice by $91 \%$ (Kenny et al., 2014) and positive self-talk by 65\% of musicians (Kenny et al., 2014).

Alexander Technique significantly improved MPA compared to a no treatment control group (Valentine et al., 1995).

Psychodramatic treatment was described as helpful by the author of a case report (Moreno, 1946).

A seminar with video feedback, body awareness and cognitive strategies resulted in a significant improvement of MPA and rated performance compared to a no treatment control group (Spahn et al., 2016).

\subsubsection{Studies with musicians not suffering from MPA}

The four studies with participants being mostly free from MPA, examined influence of benzodiazepine, beta blockers and biofeedback on anxiety during performances. 
The benzodiazepine diazepam ( $2 \mathrm{mg}$ administered 1 hour before a performance) had no significant influence on anxiety (self- and observer ratings during performances) compared to placebo (James and Savage, 1984).

In contrast two studies examining beta blockers showed some effect on anxiety: nadolol (40 mg administered 4 hours before a performance) resulted in a better bow control in string players and a significantly lower pulse rate during performance than placebo. Other observer ratings and self-ratings of performances did not differ between nadolol and placebo (James and Savage, 1984). Oxprenolol (40 mg administered 90 minutes before performance) significantly reduced self-reported anxiety ratings, pulse rate and blood pressure during performance compared to placebo (James et al., 1977, Pearson and Simpson, 1978).

For low frequency heart rate variability (HRV) biofeedback plus slow breathing no significant differences could be found in anxiety compared to reading or just slow breathing. Taking both slow breathing groups together, high frequency HRV and low frequency / high frequency ratio improved significantly during anxious anticipation compared to reading. Only for highly anxious participants slow breathing groups reduced anxiety significantly compared to the control group (Wells et al., 2012).

\section{Discussion}

The results of the present review impressively demonstrated that research on MPA currently suffered from certain methodological weaknesses and is characterized by a high degree of heterogeneity.

First, study designs, term usage and surveyed result parameters differed widely. Mainly the terms MPA, performance anxiety or stage fright were used, without clarifying what exactly was meant by these terms. Usage ranged from some excitement while being on stage up to clinically relevant MPA diagnosis. A definition of MPA according to the criteria of the established diagnostic classification systems (ICD-10, DSM-IV or DSM-5) however, did not take place in any study. Therefore, it often remained unclear what was exactly measured and a direct comparison of studies was not possible. 
All studies showed methodological deficiencies, as it is reflected in quality ratings. Especially the selection of participants was problematic. Some studies did not report diagnostic inclusion criteria and if musicians suffered from MPA or if healthy musicians were examined. It seems to be essential to first screen musicians with standardized instruments (for example: IDCL-Checklists (Hiller et al., 1994), SKID-I and II (Fydrich et al., 1997, Wittchen et al., 1997) or Composite International Diagnostic Interview (Robins et al., 1988)) and report if the sample suffered from MPA, as defined in the ICD-10, DSM-IV or DSM-5. A criteria-based sample is needed to investigate prevalence, risk factors or treatment options. Moreover, valid instruments are needed to assess disorder-specific symptom severity. In the past, unspecific measurements of anxiety like the STAI-T or STAI-S (Spielberger et al., 1982) or self-developed questionnaires were used to assess (changes in) MPA, making it impossible to interpret results or compare outcomes to other studies. There are disorder-specific instruments available measuring MPA (like K-MPAI (Kenny, 2009), Performance Anxiety Questionnaire (PAQ) (Cox and Kenardy, 1993) or PAI (Nagel et al., 1981)) with the K-MPAI being validated (Chang et al., 2018). For German studies, there is a validated German version of the PAQ available: the "Bühnenangstfragebogen" by Fehm et al. (2002).

Regarding intervention studies quality of methods was limited due to several reasons: Often participants were not randomised to different groups or could choose between different interventions or waiting list. Interventions were only rarely blinded making it possible for participants to expect some interventions, especially in comparison to waiting list controls, to be more effective. There is a need for randomised controlled trials with active control groups to research treatments for MPA. Furthermore, there should be no parallel treatments directly prior to or during the study period and comorbidities should be assessed and reported.

It was tried to derive some statements regarding prevalence, risk factors and treatment of MPA. Prevalence rate ranged from $16.5-60 \%$ and was mainly calculated by selfreports of musicians without any third-party assessment. When looking at those reports indicating MPA to be a severe problem for musicians and thus making it more possible to be clinically relevant, about one third of the musicians seem to suffer from MPA. This goes in line with anxiety disorders being the most prevalent psychiatric disorders in Europe (Wittchen et al., 2011). 
Regarding risk factors most studies reported women to be more affected than men, like it is the case with other anxiety disorders like agoraphobia, panic disorder, generalized anxiety disorder, specific phobia or social anxiety disorder (Wittchen et al., 2011), indicating some greater vulnerability of women for anxiety disorders. Concerning age, the majority of studies reported older musicians to be less affected by MPA. The reason for that might be that very anxious musicians end their career due to MPA and engage in other professions. A further reason for this finding might be some kind of adaptation to the stressing factors of performances, making it easier to deal with those, when musicians have more experience. Younger musicians are more frequently exposed to uncertain situations (vulnerabilities) when being confronted with puberty, career entry or financial uncertainties. Compared to other anxiety disorders the same pattern is visible. Older people show lower prevalence rates of anxiety disorders (Bandelow \& Michaelis, 2015). Other risk factors should be systematically investigated, like type of orchestra or the position (solo / tutti) within the instrument group. There was no study examining risk factors in an appropriate way. Most findings were surveyed with cross-sectional studies and correlations. To receive more information about risk factors there is a need for longitudinal studies recording influencing factors and presence of MPA.

Concerning treatment, the majority of studies examined cognitive behavioural therapy (CBT). Different CBT techniques were investigated (ACT, cognitive therapy, virtual reality exposure, systematic desensitization) and all resulted in reductions of MPA after interventions. This goes in line with positive effects of CBT in other anxiety disorders (Bandelow et al., 2014). For future studies, it is important to compare CBT to active control groups (instead of waitlist or no control group) to better determine its efficacy as a treatment for MPA. Bandelow et al. (2015) showed that waitlists used as control group are less effective than a psychological placebo. Therefore, it is important to use an adequate (active) control group. An effective pharmacological treatment option only for vegetative symptoms of MPA were beta blockers. Beta blockers reduced physiological symptoms of MPA, like heart rate and tremor, but anxiety, negative cognitions and behaviour were not affected. Effects for music therapy improvisation and desensitization seem to be promising, but there is need for further investigations in this field. A few studies examined effects of yoga and relaxation on MPA, but mostly effects were not better than results of control groups, with exception of one study (Sweeney and Horan, 1982). For other interventions (psychodynamic therapy, 
Alexander technique or hypnotherapy) more research is needed to clarify if weak evidence for positive effects on MPA can be confirmed.

This review focuses on performance anxiety in musicians. In fact, there are other performing groups suffering of performance anxiety like dancers or athletes. Nevertheless, we decided to focus on musicians in order to not mix up different aspects and to gain specific results for this subgroup of interest.

Strengths and limitations

For several reasons the results of the present review should be interpreted with caution. First, included studies used no consistent definition of music performance anxiety. Second, criteria for the evaluation of studies changed in the past decades. In the present systematic review the most recent update was used to develop quality assessment tools. Thereby older studies were evaluated with probably stricter criteria than applicable by the time of publication of the studies. This might have resulted in an evaluation of those studies, being too strict. Third, many included studies showed methodological weakness limiting their informative value.

A strength of the present review is that a systematic and comprehensive search of literature was carried out resulting in an update of published studies regarding performance anxiety. Furthermore, all studies were evaluated with a quality rating, making it possible to determine methodological power of each study. In the field of MPA, it is the first time that systematic quality ratings were applied.

\section{Conclusion}

Statements regarding prevalence, risk factors and treatment of MPA are limited. It is mostly unclear which criteria were used to diagnose MPA. A definition of the disease, a consistent terminology and use of validated measurement instruments are essential for further research. Diagnostic uncertainty may explain the wide range of prevalence rates. Age and gender may be identified as risk factors and there is some evidence for effective treatments of MPA (especially for CBT and regarding vegetative symptoms also beta blockers). Cross-sectional studies, cohort studies and randomised controlled trials with clear diagnostic inclusion criteria and larger samples are needed in order to address a number of outstanding issues in this area of research. 


\section{References}

Abilgaard, P. (2007). Der klinische Fall: „1,4 Promille beim Schlussapplaus“ Alkohol als Selbstmedikation bei Bühnenangst. Musikphysiologie und Musikermedizin 14.

Alpert, R. \& Haber, R. N. (1960). Anxiety in academic achievement situations. Journal of Abnormal and Social Psychology 61, 207-215.

American Psychiatric Association (2013). Diagnostic and Statistical Manual of Mental Disorders American Psychiatric Association: Arlington, VA.

Appel, S. S. (1976). Modifying solo performance anxiety in adult pianists. Journal of Music Therapy 13, 3-15.

Bandelow, B., Lichte, T., Rudolf, S., Wiltink, J. \& Beutel, E. M. (2014). The Diagnosis of and Treatment Recommendations for Anxiety Disorders. Deutsches Ärzteblatt International 111, 473-480.

Bandelow, B. \& Michaelis, S. (2015). Epidemiology of anxiety disorders in the $21^{\text {st }}$ century. Dialogues in Clinical Neuroscience 17, 327-335.

Bandelow, B., Michaelis, S. \& Wedekind, D. (2017). Treatment of anxiety disorders. Dialogues in Clinical Neuroscience 19, 93-107.

Beck, A.T. \& Steer, R.A. (1993). Beck Anxiety Inventory Manual. Psychological Corporation, San Antonio, TX.

Bissonnette, J., Dube, F., Provencher, M. D. \& Moreno Sala, M. T. (2015). Virtual Reality Exposure Training for Musicians: Its Effect on Performance Anxiety and Quality. Medical Problems of Performing Artists 30, 169-77.

Borkenau, P. \& Ostendorf, F. (2008). NEO-Fünf-Faktoren-Inventar nach Costa und Mc Crae. Hogrefe.

Brandner, K. (2001). Lampenfieber und Aufführungsangst als Stressphänomene in der künstlerischen Praxis - Eine theoretische und empirische Studie am Beispiel jugendlicher Orchestermusiker. Unveröffentlichte Master-Arbeit. Institut der Goethe-Universität Frankfurt am Main.

Brodsky, W. \& Sloboda, J. A. (1997). Clinical trial of a music generated vibrotactile therapeutic environment for musicians: Main effects and outcome differences between therapy subgroups. Journal of Music Therapy 34, 2-32.

Brugués, A. O. (2011). Music Performance Anxiety - Part 2: A Review of Treatment Options. Medical Problems of Performing Artists 26, 164-171.

Chang, A., Kenny, D. \& Burga, A. (2018). Validation of the Kenny Music Performance Anxiety Inventory (K-MPAI): A cross-cultural confirmation of its factorial structure. Psychology of Music, 46, 551-567.

Chang, J. C., Midlarsky, E. \& Lin, P. (2003). Effects of Meditation on Music Performance Anxiety. Medical Problems of Performing Artists 18, 126-130.

Cox, W. J. \& Kenardy, J. (1993). Performance Anxiety, Social Phobia, and Setting Effects in Instrumental Music Students. Journal of Anxiety Disorders 7, 49-60.

Craske, M. C., Craig, K. D. \& Kendrick, M. J. (1988). The Music Performance Anxiety SelfStatement Scale. In Dictionary of behavioral assessment techniques (ed. M. Hersen and A. S. Bellack). Pergamon: Oxforf.

Critical Appraisal Skills Programme (CASP) (2013). CASP Checklists. http://www.caspuk.net/ - !casp-tools-checklists/c18f8.

Dilling, H. \& Freyberger, H. J. (2017). Taschenführer zur ICD-10-Klassifikation psychischer Störungen. Hans Huber: Bern.

Educational Testing Service (1998). ARTS Propel Handbook for Music. Princeton, NJ: Division of Teaching, Learning, Educational Testing Service.

Fehm, L., Hille, C. \& Becker, E. (2002). Der Bühnenangstfragebogen (BAF).

Unveröffentlichtes Manuskript: Technische Universität Dresden.

Fishbein, M., Middlestadt, S. E., Ottani, V., Straus, S. \& Ellis, A. (1988). Medical

Problems Among ICSOM Musicians: Overview of a National Survey. Medical Problems of

Performing Artists 3, 1-9. 
Fydrich, T., Renneberg, B., Schmitz, B. \& Wittchen, H. U. (1997). SKID-II Strukturiertes Klinisches Interview für DSM-IV Achse II: Persönlichkeitsstörungen. Hogrefe: Göttingen.

Gates, G. A. \& Montalbo, P. J. (1987). The effect of low-dose beta-blockade on performance anxiety in singers. Journal of Voice 1, 105-108.

Gates, G. A., Saegert, J., Wilson, N., Johnson, L., Shepherd, A. \& Hearne, E. M., 3rd (1985). Effect of beta blockade on singing performance. Ann Otol Rhinol Laryngol 94, 570-4. Green, S. \& Higgins, J. P. T. (2011). Chapter 2: Preparing a Cochrane review. In Cochrane Handbook for Systematic Reviews of Interventions (ed. J. P. T. Higgins and S. Green). The Cochrane Collaboration.

Hildebrandt, H., Nubling, M. \& Candia, V. (2012). Increment of fatigue, depression, and stage fright during the first year of high-level education in music students. Medical Problems of Performing Artists 27, 43-8.

Hiller, W., Zaudig, M. \& Mombour, W. (1994). IDCL für ICD-10 - ICD-10 Checklisten. Hans Huber: Bern.

Hiner, S. L., Brandt, K. D., Katz, B. P., French, R. N. \& Beczkiewicz, T. J. (1987). Performance-Related Medical Problems Among Premier Violinists. Medical Problems of Performing Artists 2, 67-71.

Hinz, A. (2005). Kardiovaskuläre und psychische Reaktivität bei Musikern: Eine Interventionsstudie zur Podiumsangst. Musikphysiologie und Musikermedizin 12.

Hodapp, V., Langendörfer, F. \& Bongard, S. (2009). Arbeitsbedingungen, gesundheitliche Beschwerden und Aufführungsängste bei professionellen Orchestermusikern

Musikphysiologie und Musikermedizin 15.

Husek, T. R. \& Alexander, S. (1963). The effectiveness of the Anxiety Differential in examination stress situations. Educational and Psychological Measurement 23, 309-318. James, I. \& Savage, I. (1984). Beneficial effect of nadolol on anxiety-induced disturbances of performance in musicians: a comparison with diazepam and placebo. American Heart Journal 108, 1150-5.

James, I. M., Griffith, D. N., Pearson, R. M. \& Newbury, P. (1977). Effect of oxprenolol on stage-fright in musicians. Lancet 2, 952-4.

Juncos, D. G., Heinrichs, G. A., Towle, P., Duffy, K., Grand, S. M., Morgan, M. C., Smith, J. D. \& Kalkus, E. (2017). Acceptance and Commitment Therapy for the Treatment of Music Performance Anxiety: A Pilot Study with Student Vocalists. Frontiers in Psychology 8, 986. Juncos, D. G. \& Markman, E. J. (2016). Acceptance and Commitment Therapy for the treatment of music performance anxiety: A single subject design with a university student. Psychology of Music 44, 935-952.

Kenny, D., Driscoll, T. \& Ackermann, B. (2014). Psychological well-being in professional orchestral musicians in Australia: A descriptive population study. Psychology of Music 42 , 210-232.

Kenny, D. T. (2005). A systematic review of treatments for music performance anxiety. Anxiety Stress and Coping 18, 183-208.

Kenny, D. T. (2009). The factor structure of the revised Kenny music performance anxiety inventory. In The International Symposium on Performance Science (ed. A. Williamon, S. Pretty and R. Buck), pp. 37-41. European Association of Conservatories: Utrecht.

Kenny, D. T. (2011). The Psychology of Music Performance Anxiety. Oxford University Press Inc.: New York. p.48ff., p.64ff. and p.109-166.

Kenny, D. T., Arthey, S. \& Abbass, A. (2016). Identifying attachment ruptures underlying severe music performance anxiety in a professional musician undertaking an assessment and trial therapy of Intensive Short-Term Dynamic Psychotherapy (ISTDP). SpringerPlus 5, 1591.

Kenny, D. T., Davis, P. \& Oates, J. (2004). Music performance anxiety and occupational stress amongst opera chorus artists and their relationship with state and trait anxiety and perfectionism. Journal of Anxiety Disorders 18, 757-77.

Khalsa, S. B. \& Cope, S. (2006). Effects of a yoga lifestyle intervention on performancerelated characteristics of musicians: a preliminary study. Medical Science Monitor 12 , CR325-31. 
Khalsa, S. B., Shorter, S. M., Cope, S., Wyshak, G. \& Sklar, E. (2009). Yoga ameliorates performance anxiety and mood disturbance in young professional musicians. Applied Psychophysiology and Biofeedback 34, 279-89.

Kim, Y. (2005). Combined treatment of improvisation and desensitization to alleviate music performance anxiety in female college pianists: A pilot study. Medical Problems of Performing Artists 20, 17-24.

Kim, Y. (2008). The effect of improvisation-assisted desensitization, and music-assisted progressive muscle relaxation and imagery on reducing pianists' music performance anxiety. Journal of Music Therapy 45, 165-91.

Krawehl, I. \& Altenmüller, E. (2000). Lampenfieber unter Musikstudenten: Häufigkeit, Ausprägung und "heimliche Theorien". Musikphysiologie und Musikermedizin 7.

Lazarus, A. A. \& Abramovitz, A. (2004). A multimodal behavioral approach to performance anxiety. Journal of Clinical Psychology 60, 831-40.

Lehrer, P. M., Goldman, N. S. \& Strommen, E. F. (1990). A pricipal components assessment of performance anxiety among musicians. Medical Problems of Performing Artists 5.

Liberati, A., Altman, D. G., Tetzlaff, J., Mulrow, C., Gotzsche, P. C., loannidis, J. P., Clarke, M., Devereaux, P. J., Kleijnen, J. \& Moher, D. (2009). The PRISMA statement for reporting systematic reviews and meta-analyses of studies that evaluate health care interventions: explanation and elaboration. PLoS Medicine 6, e1000100.

Middlestadt, S. E. (1990). Medical Problems of Symphony-Orchestra Musicians - from Counting People with Problems to Evaluating Interventions. Revista Interamericana De Psicologia 24, 159-172.

Mills, J. (1987). Assesment of solo musical performance: A preliminary study. Bulletin of the Council for Research in Music Education 25, 119-125.

Medeiros Barbar, A. E., de Souza Crippa, J. A. \& de Lima Osório, F. (2014). Performance anxiety in Brazilian musicians: Prevalence and association with psychopathology indicators. Journal of Affective Disorders 152-154, 381-386.

Moher, D., Liberati, A., Tetzlaff, J., Altman, D. G. \& The PRISMA Group (2009). Preferred reporting items for systematic reviews and meta-analyses: the PRISMA statement. PLoS Medicine 6, e1000097.

Montello, L., Coons, E. E. \& Kantor, J. (1990). The Use of Group Music-Therapy as a Treatment for Musical Performance Stress. Medical Problems of Performing Artists 5, 49-57. Moreno, J. L. (1946). Psychodramatic treatment of performance neurosis. In Psychodrama, first volume., pp. 285-314. Beacon House: New York, NY, US.

Müller, W. E. \& Basler, H. D. (1993). Kurzfragebogen zur aktuellen Beanspruchung (KAB). Beltz: Weinheim.

Nagel, J., Himle, D. \& Papsdorf, J. D. (1981). Coping with performance anxiety. NATS Bulletin 37, 26-33.

Nagel, J. J. (2010). Treatment of music performance anxiety via psychological approaches: a review of selected CBT and psychodynamic literature. Medical Problems of Performing Artists 25, 141-8.

Nagel, J. J., Himle, D. P. \& Papsdorf, J. D. (1989). Cognitive-behavioural treatment of musical performance anxiety. Psychology of Music 17, $12-21$.

National Heart Lung and Blood Institute (Last Updated April 2014). Study Quality Assessment Tools. http://www.nhlbi.nih.gov/health-pro/guidelines/in-develop/cardiovascularrisk-reduction/tools/.

Norton, G. R., MacLean, L. \& Wachna, E. (1978). The use of cognitive desensitization and self-directed mastery training for treating stage fright. Cognitive Therapy and Research 2, 6164.

Nowlis, V. (1966). Research with the Mood Adjective Checklist. In Affect, Cognition and Personality (ed. S. S. Tomkins and C. E. Izard). Tavistock Publications: London.

Pearson, R. M. \& Simpson, A. F. (1978). Effect of oxprenolol in stage fright in musicians. Transactions of the Medical Society of London 95, 46-53.

Rapee, R. M., Craske, M. G., Brown, T. A. \& Barlow, D. H. (1996). Measurement of perceived control over anxiety-related events. Behavior Therapy 27, 279-293. 
Reiss, S., Peterson, R.A., Gursky, D.M. \& McNally, R.J. (1986). Anxiety sensitivity, anxiety frequency and the prediction of fearfulness. Behaviour. Research and Therapy. 24 (1), 1-8. Rider, M. S. (1987). Music therapy: Therapy for debilitated musicians. Music Therapy Perspectives 4, 40-43.

Robins, L. N., Wing, J., Wittchen, H. U., Helzer, J. E., Babor, T. F., Burke, J., Farmer, A., Jablenski, A., Pickens, R., Regier, D. A. \& et al. (1988). The Composite International Diagnostic Interview. An epidemiologic Instrument suitable for use in conjunction with different diagnostic systems and in different cultures. Archives of General Psychiatry 45, 1069-77.

Safirstein, S. L. (1962). Stage fright in a musician. A segment of an analysis. American Journal of Psychoanalysis 22, 15-42.

Salmon, P. G. (1992). Performance anxiety. Comprehensive casebook of cognitive therapy. (ed. A. Freeman and F. M. Dattilio), pp. 61-69. Plenum Press: New York, NY, US.

Schröder, H. \& Liebelt, P. (1999). Psychologische Phänomen- und Bedingungsanalysen zur Podiumsangst von Studierenden an Musikhochschulen. Musikphysiologie und Musikermedizin 6, 1-6.

Schwarzer, R. \& Jerusalem, M. (1999). Skalen zur Erfassung von Lehrer- und Schülermerkmalen. Dokumentation der psychometrischen Verfahren im Rahmen der Wissenschaftlichen Begleitung des Modellversuchs Selbstwirksame Schulen. Freie Universität Berlin: Berlin.

Scottish Intercollegiate Guidelines Network SIGN Methodology Checklists. Last modified September 2015. http://www.sign.ac.uk/methodology/checklists.html.

Sousa, C. M., Machado, J. P., Greten, H. J. \& Coimbra, D. (2016). Occupational Diseases of Professional Orchestra Musicians from Northern Portugal: A Descriptive Study. Medical Problems of Performing Artists 31, 8-12.

Spahn, C., Nusseck, M. \& Walther, J. C. (2013). Fragebogen zum Auftritt (Fremdeinschätzung): FZA-F, [Questionnaire for Performances (assessment of others)]. Freiburg Institute of Musicians' Medicine

Spahn, C., Richter, B. \& Altenmüller, E. (2011). MusikerMedizin: Diagnostik, Therapie und Prävention von musikerspezifischen Erkrankungen. Schattauer Verlag. p. 153.

Spahn, C., Walther, J.-C. \& Nusseck, M. (2016). The effectiveness of a multimodal concept of audition training for music students in coping with music performance anxiety. Psychology of Music 44, 893-909.

Spielberger, C. D., Gorssuch, R. L., Lushene, P. R., Vagg, P. R. \& Jacobs, G. A. (1982). Manual for the State-Trait Anxiety Inventory. Consulting Psychologists Press: Palo Alto, CA. Stanton, H. E. (1993). Research Note: Alleviation of Performance Anxiety through Hypnotherapy. Psychology of Music 21, 78-82.

Stanton, H. E. (1994). Reduction of Performance Anxiety in Music Students. Australian Psychologist 29, 124-127.

Steptoe, A. \& Fidler, H. (1987). Stage fright in orchestral musicians: a study of cognitive and behavioural strategies in performance anxiety. British Journal of Psychology 78 ( Pt 2), 2419.

Stern, J. R., Khalsa, S. B. \& Hofmann, S. G. (2012). A yoga intervention for music performance anxiety in conservatory students. Medical Problems of Performing Artists 27, 123-8.

Stern, J. R. S. (2012). Evaluation of a yoga intervention for music performance anxiety in conservatory students: A pilot study. pp. 3965-3965. ProQuest Information \& Learning: US. Sweeney, G. A. \& Horan, J. J. (1982). Separate and Combined Effects of Cue-Controlled Relaxation and Cognitive Restructuring in the Treatment of Musical Performance Anxiety. Journal of Counseling Psychology 29, 486-497.

Vaag, J., Bjoerngaard, J.H., Bjerkeset, O. (2015). Symptoms of anxiety and depression among Norwegian musicians compared to the general workforce. Psychology of Music 44(2), 234-248.

Valentine, E. R., Fitzgerald, D. F. P., Gorton, T. L., Hudson, J. A. \& Symonds, E. R. C. (1995). The Effect of Lessons in the Alexander Technique on Music Performance in High and Low Stress Situations. Psychology of Music 23, 129-141. 
van Kemenade, J. F., van Son, M. J. \& van Heesch, N. C. (1995). Performance anxiety among professional musicians in symphonic orchestras: a self-report study. Psychological Reports 77, 555-62.

Wells, R., Outhred, T., Heathers, J. A., Quintana, D. S. \& Kemp, A. H. (2012). Matter over mind: a randomised-controlled trial of single-session biofeedback training on performance anxiety and heart rate variability in musicians. PLoS One 7, e46597.

Wesner, R. B., Noyes, R. \& Davis, T. L. (1990). The Occurrence of Performance Anxiety among Musicians. Journal of Affective Disorders 18, 177-185.

Wittchen, H.U., Zaudig, M. \& Fydrich, T. (1997). Strukturiertes Klinisches Interview für DSM-IV. Hogrefe: Göttingen.

Wittchen, H. U., Jacobi, F., Rehm, J., Gustavsson, A., Svensson, M., Jonsson, B., Olesen, J., Allgulander, C., Alonso, J., Faravelli, C., Fratiglioni, L., Jennum, P., Lieb, R., Maercker, A., van Os, J., Preisig, M., Salvador-Carulla, L., Simon, R. \& Steinhausen, H. C. (2011). The size and burden of mental disorders and other disorders of the brain in Europe 2010. European Neuropsychopharmacology 21, 655-79. 


\section{Appendix 1: Search algorithms}

\section{Medline and Embase via OvidSP}

(musician $\$ 1$ OR instrumentalist $\$ 1$ OR orchestra OR symphony OR music student $\$ 1$ OR pianist $\$ 1$ OR string player $\$ 1$ OR violinist $\$ 1$ OR brass player $\$ 1$ OR cellist $\$ 1$ OR bassist $\$ 1$ OR violist $\$ 1$ OR woodwind $\$ 1$ OR flute player $\$ 1$ OR oboist $\$ 1$ OR clarinetist $\$ 1$ OR bassoonist $\$ 1$ OR hornist $\$ 1$ OR saxophonist $\$ 1$ OR brass player $\$ 1$ OR trumpet player $\$ 1$ OR bugler $\$ 1$ OR trombone player $\$ 1$ OR tuba player $\$ 1$ OR euphonium player $\$ 1$ OR harpist $\$ 1$ OR vocalist $\$ 1$ OR singer $\$ 1$ )

AND

(performance anxiety/ or performance anxiety or stage fright)

AND

(Cross-sectional study/ OR cross-sectional studies/ OR cohort studies/ OR cohort analysis/ OR case control studies/ OR case control study/ OR observational study/ OR case reports/ OR case report/ OR intervention studies/ OR intervention study/ OR exp clinical trial/ OR randomized controlled trial/ OR systematic review/ OR risk factor/OR risk factors/OR therapy/OR therapeutics/ OR exp clinical trials as topic/ OR exp "clinical trial (topic) "/ OR double-blind method/ OR double-blind procedure/ OR prevalence/ OR incidence/ OR cross-sectional stud\$ OR cohort stud\$ OR case-control-stud\$ OR observational stud $\$$ OR case report\$1 OR intervention stud\$ OR clinical trial\$1 OR double-blind method OR randomized controlled trial\$1 OR prevalence OR incidence OR systematic review $\$ 1$ OR risk factor\$1 OR treat\$ OR therap\$)

\section{Cinahl via Ebscohost}

(musician* OR instrumentalist* OR orchestra OR symphony OR music student* OR pianist* OR harpsichordist ${ }^{\star}$ OR organist ${ }^{\star}$ OR string player ${ }^{\star}$ OR violinist ${ }^{\star}$ OR brass player ${ }^{\star}$ OR cellist* OR bassist ${ }^{\star}$ OR violist OR harpist ${ }^{\star}$ OR woodwind ${ }^{*}$ OR flute player ${ }^{\star}$ OR recorder player OR oboist ${ }^{\star}$ OR clarinetist ${ }^{\star}$ OR bassoonist* OR hornist ${ }^{\star}$ OR saxophonist* OR brass player ${ }^{\star}$ OR trumpet player ${ }^{\star}$ OR bugler OR trombone player* OR tuba player* OR euphonium player* ${ }^{*}$ OR percussionist* OR drummer* OR vocalist* $^{\star}$ OR singer ${ }^{\star}$ )

AND

(performance anxiety OR stage fright)

AND

((MH "Cross Sectional Studies") OR (MH "Prospective Studies+") OR (MH "Case Control Studies+") OR (MH "Nonexperimental Studies+") OR (MH "Case Studies") OR (MH "Experimental Studies+") OR (MH "Systematic Review") OR (MH "Risk Factors") OR (MH "Clinical Trials+") OR (MH "Double-Blind Studies") OR (MH "Prevalence") OR (MH "Incidence")OR Cross-sectional stud* OR cross-sectional stud $^{*}$ OR cohort stud ${ }^{*}$ OR case-control stud* OR observational stud ${ }^{*}$ OR case report* OR intervention stud $^{*}$ OR clinical trial* OR double-blind-method OR prevalence OR incidence OR randomized controlled trial ${ }^{*}$ OR systematic review* ${ }^{*}$ risk factor ${ }^{*}$ OR treat ${ }^{*}$ OR therap*) 


\section{Psyclnfo and PsycArticles via Ebscohost}

(musician* OR instrumentalist ${ }^{\star}$ OR orchestra OR symphony OR music student ${ }^{\star}$ OR pianist* OR harpsichordist* OR organist ${ }^{\star}$ OR string player ${ }^{\star}$ OR violinist ${ }^{\star}$ OR brass player ${ }^{\star}$ OR cellist ${ }^{\star}$ OR bassist* OR violist OR harpist ${ }^{*}$ OR woodwind ${ }^{*}$ OR flute player ${ }^{*}$ OR recorder player OR oboist ${ }^{*}$ OR clarinetist ${ }^{*}$ OR bassoonist ${ }^{\star}$ OR hornist ${ }^{\star}$ OR saxophonist* OR brass player ${ }^{\star}$ OR trumpet player ${ }^{\star}$ OR bugler OR trombone player* OR tuba player* OR euphonium player ${ }^{\star}$ OR percussionist* ${ }^{*}$ OR drummer ${ }^{\star}$ OR vocalist $^{*}$ OR singer ${ }^{\star}$ )

AND

((DE "Performance Anxiety") OR performance anxiety OR stage fright)

AND

((DE "Cohort Analysis") OR (DE "Case Report") OR (DE "Clinical Trials") OR (DE "Risk Factors") OR (DE "Alternative Medicine" OR DE "Medical Treatment (General)" OR DE "Physical Treatment Methods" OR DE "Relaxation Therapy") OR (DE "Treatment") OR (DE "Clinical Trials")OR Crosssectional stud ${ }^{*}$ OR cross-sectional stud ${ }^{*}$ OR cohort stud ${ }^{*}$ OR case-control stud* OR observational stud $^{*}$ OR case report* OR intervention stud ${ }^{*}$ OR clinical trial ${ }^{*}$ OR double-blind method OR prevalence OR incidence OR randomized controlled trial ${ }^{*}$ OR systematic review* ${ }^{*}$ OR risk factor ${ }^{*}$ OR treat ${ }^{*}$ OR therap*)

\section{ERIC via Ebscohost}

(musician* OR instrumentalist* OR orchestra OR symphony OR music student* OR pianist* OR harpsichordist* OR organist ${ }^{\star}$ OR string player ${ }^{\star}$ OR violinist* OR brass player ${ }^{\star}$ OR cellist ${ }^{\star}$ OR bassist* OR violist OR harpist* OR woodwind ${ }^{*}$ OR flute player ${ }^{*}$ OR recorder player OR oboist ${ }^{\star}$ OR clarinetist* OR bassoonist* OR hornist ${ }^{\star}$ OR saxophonist* OR brass player ${ }^{\star}$ OR trumpet player ${ }^{\star}$ OR bugler OR trombone player ${ }^{\star}$ OR tuba player ${ }^{\star}$ OR euphonium player ${ }^{\star}$ OR percussionist ${ }^{\star}$ OR drummer ${ }^{\star}$ OR vocalist $^{\star}$ OR singer*)

\section{AND}

(performance anxiety OR stage fright)

\section{AND}

((DE "Incidence") OR Cross-sectional stud* OR cross-sectional stud* OR cohort stud* OR case-control stud $^{*}$ OR observational stud* OR case report* OR intervention stud ${ }^{*}$ OR clinical trial* OR double-blind method OR prevalence OR incidence OR randomized controlled trial* ${ }^{*}$ OR systematic review* $O R$ risk factor* OR treat ${ }^{\star}$ OR therap*) 


\section{Appendix 2: Quality Assessment Tool Observational Cohort and Cross-Sectional Studies}

\begin{tabular}{|c|c|c|c|c|}
\hline \multicolumn{2}{|c|}{ Rater Initia Is and Rater Number (\#1 or \#2): } & & & \\
\hline \multicolumn{5}{|c|}{ Study identific a tion (Author, Tite, Year of Public a tion, Joumal Tite): } \\
\hline & Criteria & Yes & No & Not a pplic able \\
\hline 1. & Did the authors use an appropiate method to answer their question? (i.e., the right study design) & & & \\
\hline 2. & Was the research question or objective in this paper clearly stated? & & & \\
\hline 3. & Was the study population clearly specified and definded? & & & \\
\hline 4. & Was the participation rate of eligible persons at least $50 \%$ ? & & & \\
\hline 5. & $\begin{array}{l}\text { Were all the subjects selected or recruited from the same or similar populations (including the same time period)? Were inclusion and exclusion } \\
\text { criteria for being in the study prespecified and applied uniformly to all participants? }\end{array}$ & & & \\
\hline 6. & Was a sample size justification, power description, or variance and effect estimates provided? & & & \\
\hline 7. & For the analyses in this paper, were the exposure(s) of interest measured prior to the outcome(s) being measured? & & & \\
\hline 8. & Was the timeframe sufficient so that one could reasonably expect to see an association between exposure and outcome if it existed? & & & \\
\hline 9. & $\begin{array}{l}\text { For exposures that can vary in amount or level, did the study examine different levels of the exposure as related to the outcome (e.g., categories of } \\
\text { exposure, or exposure measured as continuous variable)? }\end{array}$ & & & \\
\hline 10. & Were the exposure measures (independent variables) clearly defined, valid, reliable, and implemented consistently across all study participants? & & & \\
\hline 11. & Was the exposure(s) assessed more than once over time? & & & \\
\hline 12. & Were the outcome measures (dependent variables) clearly defined, valid, reliable, and implemented consistently across all study participants? & & & \\
\hline 13. & Were the outcome assessors blinded to the exposure status of participants? & & & \\
\hline 14. & Was loss to follow-up after baseline $20 \%$ or less? & & & \\
\hline 15. & $\begin{array}{l}\text { Were key potential confounding variables measured and adjusted statistically for their impact on the relationship between exposure(s) and } \\
\text { outcome(s)? }\end{array}$ & & & \\
\hline \multirow[t]{2}{*}{16.} & Have confidence intervals or standard deviations/standard errors been provided? & & & \\
\hline & Quality Rating & & & \\
\hline \multicolumn{5}{|c|}{ Total Points Rater \#1: } \\
\hline \multicolumn{5}{|c|}{ Total Points Rater \#2: } \\
\hline \multicolumn{5}{|c|}{ Total Points C onsensus Dec ision: } \\
\hline Additi & IComments: & & & \\
\hline
\end{tabular}

Applic a tion: Yes: C ount +1 Point; No: Count -1 Point; Not a pllic a ble: C ount 0 Point, Not rep ortet mea ns No 


\section{Appendix 3: Quality Assessment Tool Case Control Studies}

\begin{tabular}{|c|c|c|c|c|}
\hline \multicolumn{2}{|c|}{ Rater Initia Is a nd Rater Number (\#1 or \#2): } & & & \\
\hline \multicolumn{5}{|c|}{ Study identific ation (Author, Title, Year of Public a tion, J oumal Title): } \\
\hline & Criteria & Yes & No & Not a pplic able \\
\hline 1. & Did the authors use an appropiate method to answer their question? (i.e., the right study design) & & & \\
\hline 2. & Was the research question or objective in this paper clearly stated? & & & \\
\hline 3. & Was the study population clearly specified and definded? & & & \\
\hline 4. & Did the authors include a sample size justification? & & & \\
\hline 5. & Were controls selected or recruited from the same or similar population that gave rise to the cases (including the same timeframe)? & & & \\
\hline 6. & $\begin{array}{l}\text { Were the definitions, inclusion and exclusion criteria, algorithms or processes used to identify or select cases and controls valid, reliable, and } \\
\text { implemented consistently across all study participants? }\end{array}$ & & & \\
\hline 7. & Were the cases clearly defined and differentiated from controls? & & & \\
\hline 8. & $\begin{array}{l}\text { If less than } 100 \text { percent of eligible cases and/or controls were selected for the study, were the cases and/or controls randomly selected from those } \\
\text { eligible? }\end{array}$ & & & \\
\hline 9. & Was there use of concurrent controls? & & & \\
\hline 10. & $\begin{array}{l}\text { Were the investigators able to confirm that the exposure/risk occurred prior to the development of the condition or event that defined a participant } \\
\text { as a case? }\end{array}$ & & & \\
\hline 11. & $\begin{array}{l}\text { Were the measures of exposure/risk clearly defined, valid, reliable, and implemented consistently (includingthe same time period) across all study } \\
\text { participants? }\end{array}$ & & & \\
\hline 12. & Were the assessors of exposure/risk blinded to the case or control status of participants? & & & \\
\hline 13. & $\begin{array}{l}\text { Were key potential confounding variables measured and adjusted statistically in the analyses? If matching was used, did the investigators account } \\
\text { for matching during study analysis? }\end{array}$ & & & \\
\hline \multirow[t]{2}{*}{14.} & Have confidence intervals or standard deviations/standard errors been provided? & & & \\
\hline & Quality Rating & & & \\
\hline \multicolumn{5}{|c|}{ Total Points Rater \#1: } \\
\hline \multicolumn{5}{|c|}{ Total Points Rater \#2: } \\
\hline \multicolumn{2}{|c|}{ Tota I Points C onsensus Dec ision: } & & & \\
\hline
\end{tabular}

Applic a tion: Yes: C ount +1 Point; No: C ount -1 Point; Not a pllic a ble: Count 0 Po int, Not reportet means No 


\section{Appendix 4: Quality Assessment Tool pre-post Studies without control group}

\begin{tabular}{|c|c|c|c|c|}
\hline \multicolumn{2}{|c|}{ Rater Initia Is a nd Rater Number (\#1 or \#2): } & & & \\
\hline \multicolumn{5}{|c|}{ Study id entific a tion (Author, Title, Year of Public a tion, J oumal Title): } \\
\hline & C riteria & Yes & No & $\begin{array}{c}\text { Not } \\
\text { applic able }\end{array}$ \\
\hline 1. & Did the authors use an appropiate method to answer their question? (i.e., the right study design) & & & \\
\hline 2. & Was the study question or objective clearly stated? & & & \\
\hline 3. & Were eligibility/selection criteria for the study population prespecified and clearly described? & & & \\
\hline 4. & $\begin{array}{l}\text { Were the participants in the study representative of those who would be eligible for the test/service/intervention in the general or } \\
\text { clinical population of interest? }\end{array}$ & & & \\
\hline 5. & Was the sample size sufficiently large to provide confidence in the findings? & & & \\
\hline 6. & Were study participants and providers blinded to treatment group assignment? & & & \\
\hline 7. & Was the test/service/intervention clearly described and delivered consistently across the study population? & & & \\
\hline 8. & Were the outcome measures prespecified, clearly defined, valid, reliable, and assessed consistently across all study participants? & & & \\
\hline 9. & Were the people assessing the outcomes blinded to the participants' exposures/interventions? & & & \\
\hline 10. & Was the loss to follow-up after baseline $20 \%$ or less? Were those lost to follow-up accounted for in the analysis? & & & \\
\hline 11. & $\begin{array}{l}\text { Did the statistical methods examine changes in outcome measures from before to after the intervention? Were statistical tests done that } \\
\text { provided } p \text { values for the pre-to-post changes? }\end{array}$ & & & \\
\hline 12. & $\begin{array}{l}\text { Were outcome measures of interest taken multiple times before the intervention and multiple times after the intervention (i.e., did they } \\
\text { use an interrupted time-series design)? }\end{array}$ & & & \\
\hline 13. & $\begin{array}{l}\text { If the intervention was conducted at a group level (e.g., a whole hospital, a community, etc.) did the statistical analysis take into } \\
\text { account the use of individual-level data to determine effects at the group level? }\end{array}$ & & & \\
\hline 14. & Have confidence intervals or standard deviations/standard errors been provided? & & & \\
\hline \multirow[t]{2}{*}{15.} & Was the study carried out at only one site, or if not, are results comparable for all sites? & & & \\
\hline & Q ua lity Rating & & & \\
\hline \multicolumn{5}{|c|}{ Total Points Rater \#1: } \\
\hline \multicolumn{5}{|c|}{ Total Points Ra ter \#2: } \\
\hline \multicolumn{5}{|c|}{ Total Points C onsensus Dec ision: } \\
\hline Additio & IComments: & & & \\
\hline
\end{tabular}

Applic a tion: Yes: C ount +1 Point; No: C ount -1 Point; Not a pllic a ble: C ount 0 Point; Not reportet means No 


\section{Appendix 5: Quality Assessment Tool Intervention Studies with control group}

\begin{tabular}{|c|c|c|c|c|}
\hline \multicolumn{2}{|c|}{ Rater Initia Is and Rater Number (\#1 or \#2): } & & & \\
\hline \multicolumn{5}{|c|}{ Study identific ation (Author, Title, Year of Public ation, J oumal Title): } \\
\hline & Criteria & Yes & No & Not a pplic able \\
\hline 1. & Did the authors use an appropiate method to answer their question? (i.e., the right study design) & & & \\
\hline 2. & Was the research question or objective in this paper clearly stated? & & & \\
\hline 3. & Was the study described as randomized, a randomized trial, a randomized clinical trial, or an RCT? & & & \\
\hline 4. & Was the method of randomization adequate (i.e., use of randomly generated assignment)? & & & \\
\hline 5. & Was the treatment allocation concealed (so that assignments could not be predicted)? & & & \\
\hline 6. & Were study participants and providers blinded to treatment group assignment? & & & \\
\hline 7. & Were the people assessing the outcomes blinded to the participants' group assignments? & & & \\
\hline 8. & $\begin{array}{l}\text { Were the groups similar at baseline on important characteristics that could affect outcomes (e.g., demographics, risk factors, co-morbid } \\
\text { conditions)? }\end{array}$ & & & \\
\hline 9. & Was the overall drop-out rate from the study at endpoint $20 \%$ or lower of the number allocated to treatment? & & & \\
\hline 10. & Was the differential drop-out rate (between treatment groups) at endpoint 15 percentage points or lower? & & & \\
\hline 11. & Was there high adherence to the intervention protocols for each treatment group? & & & \\
\hline 12. & Were other interventions avoided or similar in the groups (e.g., similar background treatments)? & & & \\
\hline 13. & Were outcomes assessed using valid and reliable measures, implemented consistently across all study participants? & & & \\
\hline 14. & $\begin{array}{l}\text { Did the authors report that the sample size was sufficiently large to be able to detect a difference in the main outcome between groups with at } \\
\text { least } 80 \% \text { power? }\end{array}$ & & & \\
\hline 15. & Were outcomes reported or subgroups analyzed prespecified (i.e., identified before analyses were conducted)? & & & \\
\hline 16. & Were all randomized participants analyzed in the group to which they were originally assigned, i.e., did they use an intention-to-treat analysis? & & & \\
\hline 17. & Have confidence intervals or standard deviations/standard errors been provided? & & & \\
\hline \multirow[t]{2}{*}{18.} & Was the study carried out at only one site, or if not, are results comparable for all sites? & & & \\
\hline & Qua lity Rating & & & \\
\hline \multicolumn{5}{|c|}{ Total Points Rater \#1: } \\
\hline \multicolumn{5}{|c|}{ TotaI Points Rater \#2: } \\
\hline \multicolumn{5}{|c|}{ Total Points Consensus Dec ision: } \\
\hline Additi & IComments: & & & \\
\hline
\end{tabular}

Applic a tion: Yes: Count +1 Point; No: Count -1 Point; Not a pllic a ble: C ount 0 Point; Not rep ortet mea ns No 